\title{
Beforschung von Gestalttherapie bei Angststörungen in Praxisumgebungen
}

\section{Ein experimentelles Einzelfall-Design ${ }^{1}$}

\author{
Pablo Herrera, Illia Mstibovskyi, Jan Roubal \& Philip Brownell \\ Psychotherapie-Wissenschaft 9 (2) 53-69 2019 \\ www.psychotherapie-wissenschaft.info \\ CC BY-NC-ND \\ https://doi.org/10.30820/1664-9583-2019-2-53
}

\begin{abstract}
Zusammenfassung: Trotz der nachgewiesenen Wirksamkeit von Kognitiver Verhaltenstherapie (KVT) bei generalisierten Angststörungen sprechen $33 \%$ der PatientInnen nicht auf die Therapie an und 50\% brechen diese ab. Gestalttherapie wird als wirksame Alternative bezeichnet, aber es gibt nur wenige empirische Belege für ihre Wirksamkeit bei Angststörungen. Das experimentelle Einzelfall-Design mit einer Zeitreihenanalyse wurde als praxisorientierte Wirksamkeitsstudie angewendet. Darin werden Daten von zehn Klientinnen mit einer diagnostizierten Angststörung vorgestellt, die die Behauptung stützen, dass Gestalttherapie eine nützliche Behandlung für diese Indikation sein kann. Die detaillierte Analyse eines Falls veranschaulicht die Veränderung der Scores für Symptome und Wohlbefinden, und illustriert damit Wendepunkte im Verlauf der Therapie. Das Paper behandelt die Anwendung dieser Methodologie zur Schaffung eines praxisorientierten Forschungsnetzwerks.
\end{abstract}

Schlüsselwörter: Angststörung, Gestalttherapie, Einzelfall, experimentelles Zeitreihendesign, praxisorientierte Forschung

Jahrzehnte systematischer Forschung haben die Wirksamkeit psychotherapeutischer Behandlungen, einschliesslich der Behandlung von PatientInnen mit verschiedenen Formen von Angststörungen, nachgewiesen (Roth \& Fonagy, 2013). Die Evidenzlage zur Wirksamkeit von Verhaltens- und kognitiven Verhaltenstherapien, die als Behandlung der Wahl für die meisten Angststörungen angesehen werden, ist umfassend (Hollon \& Beck, 2013). Diese Ansätze scheinen jedoch für eine beträchtliche Patientengruppe nicht ausreichend hilfreich zu sein. Nur $50 \%$ der KVT-PatientInnen mit Generalisierter Angststörung erreichen einen hohen Funktionsstatus zu Therapieabschluss, etwa $30 \%$ der PTBS-PatientInnen brechen die KVT ab und mindestens ein Drittel der PatientInnen mit sozialer Phobie spricht nicht auf kognitive Verhaltenstherapie an (Lambert, 2013). Die Suche nach alternativen evidenzbasierten Ansätzen, als Ergänzung für die prävalente Behandlung (KVT), ist ein Weg, um die Möglichkeiten der psychologischen Hilfe für PatientInnen mit Angststörung zu erweitern.

Trotz langer Erfahrung in der Arbeit mit Angststörungen, gibt es keine robusten Belege für die Wirksamkeit humanistisch-experienzieller Behandlungen von Angststörungen (Angus et al., 2015; Elliot et al., 2013). Beispielsweise kam kürzlich eine Besprechung der empirischen Belege für humanistische Therapien zu der Schlussfolgerung, dass sie bei Angststörungen weniger effektiv sind als KVT und

1 Der engl. Originalartikel erschien 2018 in Revista Argentina de Clinica Psicologica, 27(2), 321-336 (https://doi.org/10.24205/03276716.20 18.1066) und ist auf www.psychotherapie-wissenschaft.info einsehbar. dass sie nur für KlientInnen in Betracht gezogen werden sollten, bei denen bereits eine KVT ausprobiert wurde, oder die diese abgelehnt hatten (Angus et al., 2015). Obwohl die aktuellen Ergebnisse unter der negativen Forschungszuordnung leiden sowie unter einer teilweise falschen Darstellung der humanistischen Therapien (Elliot et al., 2013), scheint es so, als würden PatientInnen mit Angststörungen besser auf strukturiertere Behandlungen ansprechen und so, als seien «experienzielle Therapien bisher in dieser KlientInnenpopulation nicht effektiv angewandt worden» (ebd., S. 8). Daher ist weitere Forschung in dieser speziellen Population notwendig.

Die aktuelle Forschung über die Effektivität von humanistisch-experienziellen Therapien bei Angststörungen ist sehr begrenzt. Die meisten vorliegenden Studien thematisieren Klientenzentrierte Psychotherapie, keine die fokusorientierte Therapie, sehr wenige die sehr aktuellen offenen Studien zu Emotionsfokussierter Therapie (Shahar et al., 2017; Timulak et al., 2017; Watson \& Greenberg, 2017) und nur eine Studie beschäftigt sich mit Gestalttherapie (Elliot et al., 2013). Weitere Forschung zu diesem Thema könnte dabei helfen, die Wirksamkeit der strukturierteren humanistisch-experienziellen Modalitäten (z.B. Gestalttherapie, EFT) zu belegen, und Angststörungen von einem humanistisch-experienziellen Standpunkt aus besser zu verstehen. Wie Elliot (2013, S. 12) schlussfolgerte:

«Ich zweifele nicht daran, dass Klientenzentrierte Ansätze und experienzielle Therapien viel dazu beitragen können, KlientInnen mit Angststörungen zu helfen, vor 
allem wenn wir die Zeit und Energie investieren, um Forschung zu betreiben, die wirklich das repräsentiert, was wir tun, und wenn wir mit unseren KlientInnen zusammenarbeiten, um die Angemessenheit und Effektivität von dem zu verbessern, was wir anzubieten haben.»

\section{Angststörungen aus der Perspektive der Gestalttherapie}

Gestalttherapie ist ein phänomenologischer, existenzieller und relationaler Ansatz, der auf dem holistischen und dynamischen Organismus-Umwelt-Feld als zugrundeliegender Anthropologie basiert. Theorie und Praxis der Gestalttherapie bieten differenzierte Ansätze in vielfältigen klinischen Umgebungen mit unterschiedlichen klinischen Populationen (Francesetti et al., 2013), einschliesslich der Behandlung von Angststörungen (Robine, 2013).

Aus der Perspektive der Gestalttherapie ist Angst ein holistisch erfahrener Zustand der Erregung des Organismus, dem die Unterstützung für eine Handlung zum Ausdruck eines Bedürfnisses fehlt. Eine Angststörung wird nicht als Pathologie betrachtet, sondern eher als Zeichen dafür, dass die gesunde Erregung des Organismus unterbrochen wurde; mit angemessener therapeutischer Unterstützung könne diese jedoch wieder zum Wachstum gelenkt werden (Ceballos, 2014). Neurotische Angst entsteht, wenn bestimmte organismische Bedürfnisse als inakzeptabel angesehen und ihr Ausdruck und die damit verbundene Erregung daher systematisch von festen relationalen Mustern unterdrückt werden, die die Flexibilität und Kreativität des Potenzials des Individuums, auf verschiedene Situationen zu reagieren, auf eine Weise beschränken, durch das ihre/ seine Bedürfnisse hier und jetzt erfüllt werden würden (ebd.; Herrera, 2016). Neurotische Angst kann auch aufgelöst werden, wenn die Person im Hier und Jetzt sein kann, anstatt über die Zukunft zu fantasieren und diese zu katastrophisieren (Perls, 1969).

Die Gestalt-Perspektive ist inhärent relational. Die im Körper erfahrenen Symptome der Angststörung werden als individueller Ausdruck des relationalen Leidens verstanden (Roubal et al., 2013), wenn es dem Kontaktprozess des Individuums zu anderen Menschen an Spontaneität und Flüssigkeit mangelt. In solchen Fällen ist die Art des zwischenmenschlichen Kontakts durch feste relationale Muster gestört, und Bedürfnisse werden innerhalb von Beziehungen nicht erfüllt. Die Beziehung $\mathrm{zu}$ anderen und sich selbst durch das Bedürfnis «das Richtige zu tun» zu definieren, kann ein Beispiel für solche Störungen sein, die die Erfahrungsmöglichkeiten und kreativen Anpassungen einschränken (Robine, 2013). Der Fluss der Figur-/Grund-Bildung wird gestört, da die Person Angst hat, Risiken einzugehen, kreativere Wege zu finden, um einen für beide Seiten zufriedenstellenden Kontakt einzugehen. Auch wenn die Erregung vorhanden ist, ist der Organismus gehemmt oder sogar gelähmt.

In der Therapie wird Unterstützung benötigt, um Angst in flüssige und kreative Aufregung umzuwandeln. Die Unterstützung stammt aus der therapeutischen Beziehung, in der die Bedürfnisse der KlientInnen erkannt und validiert werden. In der sicheren Therapiesituation sind die Hemmungen der/des Klientin/en reduziert und die Erregung seines/ihres Organismus kann in den Ausdruck von Beziehungsbedürfnissen geleitet werden. Die Erfahrung einer/s Klientin/en könnte sein: «Mit der/m Therapeutin/ en kann ich es riskieren, ich selbst zu sein, ohne dies als richtig oder falsch zu bewerten.» Die lähmende Angst wird in die Freude über die Entdeckung neuer kreativer Arten des Kontakts gewandelt. Die KlientInnen experimentieren mit diesen zunächst in der sicheren psychotherapeutischen Beziehung und später auch mit anderen Menschen.

\section{Der Bedarf für alternative Methodologien bei der Wirksamkeitsforschung von Gestalttherapie}

Eine der wichtigsten Herausforderungen der empirischen Unterstützung eines Behandlungsmodells liegt in der für die Forschung verwendeten Methodologie (Borckardt et al., 2008). Das Design einer randomisierten klinischen Studie (RCT), das für Wirksamkeitsstudien angewendet wird, wird als Goldstandard angesehen, um die Kausalität zwischen Behandlung und PatientInnenergebnissen festzustellen. Es weist jedoch mehrere Schwierigkeiten auf, vor allem: (1) Es ist zu teuer und schwierig umzusetzen, da es eine Methodologie für Gruppen ist, die für viele PraktikerInnen und ForscherInnen unerreichbar ist. (2) Es erzeugt Laborbedingungen, die vom gewöhnlichen Kontext, in dem die Psychotherapie durchgeführt wird, stark abweichen. (3) Es reduziert die komplexen Realitäten und Probleme der PatientInnen auf eine diagnostische Bezeichnung. (4) Es stellt nur Ergebnisse dar, und ermöglicht ForscherInnen nicht, den am Prozess beteiligten Veränderungsprozess und die Veränderungsmechanismen zu verstehen. (5) Die Bedingung der Homogenität, die es auferlegt, und die Annahmen über KlientInnen, TherapeutInnen und Behandlungen haben zu statistischen und konzeptuellen Problemen geführt, und diese sind als das wichtigste Hindernis bei der Entwicklung von Forschung in der Psychotherapie erkannt worden (Carey \& Stiles, 2015; Silberschatz, 2017; Tschuschke et al., 2010).

Dies sind wichtige Einschränkungen für die Durchführung von Wirksamkeitsforschung zu Gestalttherapie und anderen humanistischen Ansätzen, da sich Gestalt-ForscherInnen in der Regel nicht in akademischen Positionen befinden, die es ihnen ermöglichen, Gruppenstudien mit Studierendenpopulationen durchzuführen oder die finanziellen Mittel dafür zu erhalten, und auch da zwischen der humanistischen Tradition und der reduktionistischen Art, wie in Labors geforscht wird, epistemologische Diskrepanzen bestehen (Angus et al., 2015). Angesichts dieser Realität basieren Lehre und Praxis der Gestalttherapie weiterhin hauptsächlich auf klinischen Intuitionen und Einzelberichten, die sich nicht auf empirische Forschung stützen. Daher enthalten Gesundheitsrichtlinien, die eine empirische Bestätigung erfordern, Gestalttherapie nicht als Behandlungsalternative für PatientInnen, wodurch ihnen diese potenziell nützliche Behandlungsoption vorenthalten wird. 


\section{Das Einzelfall-Zeitreihendesign}

In diesem Kontext praktischer und epistemologischer Einschränkungen hat die American Psychological Association (APA) zugestimmt, dass RTC nicht die einzige Option für die Erforschung der Wirksamkeit und als empirischer Nachweis für eine Behandlungsmethode sein sollte (APA, 2006). Sie schlägt als gültige Alternative ein Einzelfall-Zeitreihendesign vor (ebd.; Chambless et al., 1998; Chambless \& Ollendick, 2001) und gibt an, dass eine grosse Serie (>9) von experimentellen EinzelfallStudien für den Nachweis, dass eine Therapie in der Praxis gut etabliert ist, ebenso akzeptabel wäre wie zwei Experimente zwischen zwei Gruppen (Chambless et al., 1998). Auf gleiche Weise äussern Borckhardt et al. (2008, S. 77), dass das ein

«von PraktikerInnen generiertes, fallbasiertes Zeitreihendesign mit Baseline-Messung als vollwertiges Experiment bezeichnet werden kann und neben den weiter verbreiteten Gruppendesigns (z. B. randomisierte kontrollierte Studie, RCT) als praktikabler Ansatz für die Mehrung unseres Wissens darüber stehen sollte, ob, wie und für wen Psychotherapie funktioniert.»

Im Einzelfall-Zeitreihendesign wird ein Einzelfall im Verlauf studiert, entlang verschiedener Phasen vor, während und nach der psychotherapeutischen Intervention. Es ist ein Experiment, da die/der PatientIn mit sich selbst verglichen wird, mit und ohne Intervention, und bei dem Baseline-Daten zur Bewertung der Probleme der/s Patientin/en gesammelt werden (abhängige Variable), ohne Intervention (Kontrollbedingung) und später zum Vergleich Daten während und nach der Intervention (Interventionsbedingung). Daher fungiert die/der PatientIn als eigene Kontrolle. Die Methode zählt zu den Zeitreihenanalysen, da die Daten fortlaufend, mit regelmässigen täglichen Messungen, erfasst und anschliessend unter Berücksichtigung der Autokorrelation und möglicher Störfaktoren auf die Wirksamkeit, wie eine natürlich Remission aufgrund des Verstreichens von Zeit, analysiert werden.

Das Einzelfall-Zeitreihendesign ist für die Untersuchung der Wirksamkeit von Psychotherapie-Modellen vorteilhaft, für die die RCT-Methodologie nicht angewendet werden kann oder soll. Sein Nutzen wurde in mehreren Forschungsprojekten nachgewiesen, die von Behavioral Activation während stationären Aufenthalten in der Psychiatrie (Folke et al., 2015; Silberschatz, 2017) über die Dokumentierung von schulbasierter Kommunikation mit Kindern auf dem Autismus-Spektrum (Whalon et al., 2015) bis zu KVT bei gleichzeitig auftretender Angststörung und Depression (Hague et al., 2015) reichen. Das Design der vorliegenden Studie entspricht auch den $\mathrm{N}=1$-Studienreihen, gefolgt von Meta-Analysen, die im Gesundheitswesen aktuell beliebter als RCT werden (Mengersen et al., 2015; Punja et al., 2016).

Für Prozess-Ergebnis-Studien kann das EinzelfallZeitreihendesign mit qualitativen Forschungsmethoden kombiniert werden, wodurch vor, während und nach der Psychotherapie detaillierte und kontinuierliche Informationen über den Veränderungsprozess der/s Klientin/ en vorliegen. Dies kann ein Indikator dafür sein, welche Therapiesitzungen einen positiven, neutralen oder negativen Einfluss auf die Symptomatik der/s Klientin/en hatten. Und dadurch kann die Entwicklung des Veränderungsprozesses im Verlauf dargestellt werden und die Phasen und entscheidenden Wendepunkte im Veränderungsprozess können genau identifiziert werden.

Zusammenfassend gesagt stellt ein experimentelles Einzelfall-Design mit einer Zeitreihenanalyse eine akzeptable Alternative zu randomisierten kontrollierten Gruppenstudien dar (Smith, 2012; Kratochwill \& Levin, 2010) und bietet wertvolle zusätzliche Vorteile. Das Einzelfall-Zeitreihendesign ist besser zu kontrollieren und kostengünstiger als Gruppendesigns (Chambless \& Hollon, 1998). Es ist weniger invasiv, wodurch es PraxisforscherInnen ermöglicht wird, in ihrer täglichen Praxis und normalen Arbeitsweise zu forschen. Es ermöglicht eine Schlussfolgerung der Ursachen im psychotherapeutischen Veränderungsprozess.

Das Ziel dieser Studie ist es, die Wirksamkeit der Gestalttherapie bei KlientInnen mit diagnostizierter Angststörung nachzuweisen. Die Studie zeigt auch eine Anwendung des Einzelfall-Zeitreihendesigns in einem praxisbasierten Forschungsnetzwerk.

\section{Methodologischer Rahmen}

\section{Experimentelles Einzelfall-Design}

In diesem experimentellen A-B-A-Einzelfall-Design mit einer Zeitreihenanalyse gibt es drei Phasen: (1) eine anfängliche Baseline-Phase ohne Therapie (zwei Wochen, beginnend mit einer Einstufungssitzung « 0 » und endend mit der ersten Therapiesitzung), (2) eine Therapiephase (mindestens acht Gestalttherapie-Sitzungen mit der maximalen Länge, abhängig vom individuellen Fall) und (3) eine Nachbeobachtungsphase (zwei Wochen, beginnend mit der letzten Therapiesitzung).

\section{Messungen}

Das Mini International Neuropsychiatric Interview (MINI 6.0; Sheehan et al., 1998) ist ein kurzes strukturiertes Interview zur Bewertung von 17 häufigen Achse-I-Störungen mittels der DSM-IV-Kriterien. Es wurde von PsychotherapeutInnen oder PsychiaterInnen mit DSM-IV-Ausbildung durchgeführt, um die Diagnose der KlientInnen zu bestätigen.

Die Hamilton-Anxiety-Skala (Hamilton, 1959) ist ein strukturiertes Interview zur Bewertung der aktuellen somatischen und psychischen Angststörung der KlientInnen. Sie verwendet folgende Grenzwerte: (1) 0-5: keine Angststörung, (2) 6-14: leichte Angststörung, (3) 15-30: mittelschwere Angststörung, $(4) \geq 31$ : schwere Angststörung. Sie verfügt über eine gute interne Konsistenz (Cron- 
bachs Alpha $0,79-0,86)$ und Interrater-Übereinstimmung $(\mathrm{r}=0,74-0,96)$.

Der Ergebnisfragebogen (OQ-45.2; Lambert et al., 1996) ist ein kurzer Fragebogen zur Selbsteinschätzung des allgemeinen Wohlbefindens der KlientInnen. Er verfügt über drei Skalen: Symptombelastung, zwischenmenschliche Beziehungen und soziale Integration. Der Grenzwert beträgt 73 und der Reliable Change Index $(\mathrm{RCI}) \geq 17$, basierend auf der chilenischen Adaption durch von Bergen und de la Parra (2002).

Das Beck-Depressions-Inventar (BDI-1; Beck, 1978) ist ein kurzer Fragebogen zur Selbsteinschätzung, zur Bewertung der Intensität, des Schweregrads und der Stärke der Depression. Die Grenzwerte betragen jeweils (1) 0-9: klinisch unauffällige Depression, (2) 10-18: milde Depression, (3) 19-29: mittlere Depression, (4) 30-63: schwere Depression.

Die Target Complaints Scale (Battle et al., 1966) ist eine individualisierte Selbsteinschätzung zur Messung von drei bis vier spezifischen idiosynkratrischen Hauptbeschwerden, die von KlientInnen und TherapeutInnen in der Sitzung «0» identifiziert und festgelegt wurden. Die Punktespanne reicht von 1 bis 10 und jede Beschwerde wird individuell analysiert. Die Beschwerden sollten konkret, quantifizierbar, regelmässig, ohne Behandlung stabil und relativ unabhängig voneinander sein. Sie werden für die Zeitreihenanalyse verwendet. Die Zuverlässigkeit dieser Messung wird als angemessen hoch angesehen, aber es fehlen weitere Gültigkeitsdaten (Deane et al., 1997).

Das Erfahrungstagebuch für TherapeutInnen ist eine Aufzeichnung der Erfahrungen und Notizen der TherapeutInnen über Therapie und Forschungsprozess basierend auf dem CSEP-II Experiential Therapy Session Form (Elliot, 2003), dessen Ergebnisse in diesem speziellen Aufsatz nicht verwendet werden.

\section{Teilnehmende}

Klientinnen (im Folgenden dargestellt alle zehn weiblich): Die Psychotherapie wurde in einer individuellen Umgebung durchgeführt, sowohl in Privatpraxen als auch im Kontext des öffentlichen Gesundheitssystems. Die Klientinnen mussten die folgenden Einschlusskriterien erfüllen: (1) Vorhandensein einer Angststörung laut MINI, oder ein Wert $\geq 15$ auf der Hamilton-Anxiety-Skala; (2) keine paranoiden oder psychotischen Symptome; (3) keine Beschwerden, die dringend eine psychotherapeutische Behandlung erfordern; (4) keine andere parallele Therapie für die gleichen Zielbeschwerden zwischen drei Wochen vor und drei Wochen nach der ersten Therapiesitzung. Aus ethischen Gründen war es den Teilnehmerinnen nach dieser Drei-Wochen-Frist gestattet, an anderen Therapieformen teilzunehmen. Es gab jedoch keine der Klientinnen an, nach Beginn der Psychotherapie-Sitzungen eine andere Therapieform begonnen zu haben. In Tabelle 1 ist eine detaillierte Beschreibung abgebildet.

TherapeutInnen: Jede Klientin wurde von einer/m anderen Therapeutin/en (acht weiblich, zwei männlich) behandelt. Alle TherapeutInnen waren Master-Studierende (Ausbildungsprogramm Gestalttherapie, Center of Gestalt Psychotherapy of Santiago) im dritten Ausbildungsjahr und die Teilnahme an der Studie war eine der Alternativen für ihre Abschlussarbeit (die andere Möglichkeit war eine theoretische Arbeit). Jede/r TherapeutIn musste die folgenden Einschlusskriterien erfüllen: (1) mindestens fünf Jahre eines Bachelor-Studiums in Psychologie, Soziale Arbeit oder Psychiatrie, (2) mindestens zwei Jahre (mindestens 360 Stunden) Graduiertenausbildung in Gestalttherapie, (3) für die Dauer der Studie Zugang zu Supervision mit in Gestalttherapie geschulten SupervisorInnen.

Übereinstimmung mit dem Behandlungsverfahren (treatment fidelity): Diese basierte auf der Psychotherapie-

\begin{tabular}{|r|r|l|l|c|}
\hline $\begin{array}{c}\text { Patient- } \\
\text { innen- } \\
\text { Nr. }\end{array}$ & Alter & \multicolumn{1}{|c|}{ Diagnose } & \multicolumn{1}{|c|}{ Hamilton-Score } & $\begin{array}{c}\text { Anzahl der } \\
\text { Sitzungen }\end{array}$ \\
\hline 1 & 39 & Angststörung & 13 (leicht) & 14 \\
\hline 2 & 30 & Generalisierte Angststörung & 21 (mittelschwer) & 15 \\
\hline 3 & 23 & Angststörung & 17 (mittelschwer) & 12 \\
\hline 4 & 26 & Alkoholmissbrauch, Agoraphobie, Depression & 19 (mittelschwer) & 18 \\
\hline 5 & 23 & Panikstörung, Agoraphobie, Generalisierte Angststörung & 29 (mittelschwer) & 40 \\
\hline 6 & 29 & Angst und depressive Störung, gemischt & 17 (mittelschwer) & 19 \\
\hline 7 & 37 & Angst und depressive Störung, gemischt & 39 (schwer) & 8 \\
\hline 8 & 24 & Angststörung & 25 (mittelschwer) & 11 \\
\hline 9 & 24 & Anpassungsstörung mit Angstsymptomen & 23 (mittelschwer) & 24 (mittelschwer) \\
\hline 10 & 26 & Panikstörung ohne Agoraphobie & & 16 \\
\hline
\end{tabular}

\section{Tab. 1: Beschreibung der Stichprobe}


Ausbildung und der Supervision der TherapeutInnen, die beide in Gestalttherapie-Modalität durchgeführt wurden.

Das Auswablverfahren: Klientinnen, die die Auswahlkriterien erfüllten, wurden von der/m jeweiligen Therapeutin/en über Telefon oder E-Mail kontaktiert und zur Teilnahme an der Studie eingeladen. Die ersten zehn Fälle, die mindestens acht Therapiesitzungen absolvierten, wurden für diese Studie ausgewählt. Nach Erfassung der Daten für diese zehn Fälle berichtete ein weiterer Therapeut über einen Klienten, der weniger als acht Sitzungen wahrgenommen und die Therapie abgebrochen hatte. Dieser wurde nicht in die Studie aufgenommen.

Ethik: Vor der Eingangseinstufung in Sitzung 0 wurden die Klientinnen über das allgemeine Studiendesign und seine Bedeutung informiert und unterzeichneten in Sitzung 0 eine Einverständniserklärung. Alle Daten wurden in Übereinstimmung mit den Richtlinien der Universidad de Chile anonymisiert gespeichert.

\section{Datenerhebung}

Baseline-Phase (A): In Sitzung 0 wendeten die TherapeutInnen den ersten Satz von Datenerhebungsinstrumenten an (MINI 6.0., EB-45 und Hamilton-Anxiety-Skala) und die Zielbeschwerden (Target Complaints TC) wurden gemeinsam mit der Klientin identifiziert. Nach Sitzung 0 begann die Patientin täglich die Zielbeschwerden aufzuzeichnen. Nach zwei Wochen (14 Tage Aufzeichnungen) begann die Therapiephase mit der ersten psychotherapeutischen Sitzung.

Therapiephase (B): Die Aufzeichnung der täglichen Beschwerden wurde fortgesetzt. Nach jeder Sitzung füllten die TherapeutInnen das Erfahrungstagebuch für TherapeutInnen aus und die Patientinnen den EB-45. Von allen Sitzungen wurden Video- und Audio-Aufzeichnung erstellt.

Nachbeobachtungsphase (A): Die Nachbeobachtungsphase begann mit der letzten Therapiesitzung, in der der EB-45 und die Hamilton-Anxiety-Skala angewendet wurden. Die Aufzeichnung der täglichen Beschwerden wurde für zwei Wochen nach der Abschlusssitzung fortgesetzt. Nach sechs Monaten kontaktierte ein/e unabhängige/r InterviewerIn die Klientin und wendete bei einem Nachbeobachtungsbesuch BDI-1, EB-45 und die HamiltonAnxiety-Skala an.

\section{Datenanalyse}

Das experimentelle Einzelfall-Design mit einer Zeitreihenanalyse (Smith, 2012) wurde angewendet, um die Werte der Zielbeschwerden (TC) der Ausgangssituation (Baseline) mit denen der nachfolgenden Phasen zu vergleichen. Die Qualitätsstandards für das EinzelfallZeitreihendesign waren wie folgt: (1) Sowohl eine visuelle als auch eine statistische Analyse (und die statistischen Instrumente für die Autokorrelation) wurden angewendet. (2) Zielbeschwerden wurden mit Standard-Ergebnis- oder
Symptom-Messungen ergänzt. (3) Therapieunabhängige Trends, die eine Besserung der Patientinnen erklären konnten, wurden kontrolliert. (4) Effektstärkedaten wurden für eine zukünftige Aggregation von multiplen Einzelfallanalysen und Meta-Analysen in Betracht gezogen (Borckardt et al., 2008; Borckardt \& Nash, 2014; Tate et al., 2013; Wendt \& Miller, 2012). Die Datenanalyse wurde zur Beantwortung der folgenden drei Fragen durchgeführt:

Forschungsfrage I: Gibt es im Vergleich vor und nach der Intervention eine Verbesserung, und falls $j a$, wie gross ist diese? Es wurden drei verschiedene Indikatoren verwendet: (1) visuelle Analyse zum Vergleich der Werte der Zielbeschwerden (TC) während der drei Phasen; (2) Prüfung von Level-Änderungen im Vergleich von der Baseline- und Nachbeobachtungsphase, mittels rKoeffizient (Pearson-Korrelationskoeffizient der TC mit Phasenvektor, $\mathrm{p}<0.05)$ zur Bewertung der Effektstärke (ES). Wie von Borckardt et al. (2008) vorgeschlagen, wurde dies mittels ihrer eigenen Software zur Analyse von Simulationsmodellen (SMA) berechnet, die unter http://clinicalresearcher.org frei zugänglich ist und speziell für eine kleine Anzahl von Beobachtungen und die Berücksichtigung der Zeitreihen-Autokorrelation entwickelt wurde; (3) die durchschnittliche Reduktion des Ausgangswertes (MBLR, berechnet, indem der durchschnittliche Nachbeobachtungswert vom durchschnittlichen Ausgangswert subtrahiert, durch den durchschnittlichen Ausgangswert dividiert und das Ergebnis mit 100 multipliziert wurde), als eine der am häufigsten berichteten und zuverlässigsten Methoden zur Berechnung der Effektstärken von Einzelfall-Designs (Campbell, 2003; Olive \& Smith, 2005).

Forschungsfrage II: Ist die Veränderung klinisch bedeutsam? Wenn es Belege einer Verbesserung im Vergleich vor und nach der Intervention gab, wurde die Analyse darauf konzentriert, wie klinisch bedeutsam die Veränderung war. Es wurden zwei Indikatoren verwendet: (1) EB-45-Werte von Sitzung 0, der Abschluss- und der Nachbeobachtungssitzung wurden unter Berücksichtigung des chilenischen Reliable Change Index von $\geq 17$ verglichen. (2) Die Werte der Hamilton-Anxiety-Skala und der BDI-Skala von Sitzung 0, der Abschluss- und der Nachbeobachtungssitzung wurden verglichen.

Forschungsfrage III: Kann die Verbesserung dem Therapieprozess zugeschrieben werden? Um nachzuweisen, dass die Besserung auf die Intervention zurückzuführen war und nicht Ergebnis eines Abwärtstrends der Zielbeschwerdenbewertungen, der mit der Baseline-Phase begann und sich einfach über die Therapie- und Nachbeobachtungsphasen fortsetzte, wurde folgende Methode eingesetzt:

1. Es wurde mithilfe der Standardmethode, der linearen Regressionsanalyse, untersucht, ob ein solcher Trend bestand: R2-Wert, p-Wert des F-Tests für die Signifikanz der Gesamtregression. Gab es keinen Trend, war die Antwort auf diese Forschungsfrage «Ja». Gab es einen Trend, dann:

2. Berechnung der ES, die sich ergibt, wenn der Einfluss des Trends beseitigt wird, mithilfe einer partiellen SMA-Korrelationsanalyse, die auf den beobachteten 
Trend hin kontrolliert wird (dieser Prozess misst dasselbe Pearson-r unter der Bedingung, dass die Korrelation der Zielbeschwerde und der lineare Trend eliminiert werden). War die daraus resultierende ES statistisch signifikant, war die Antwort auf die Forschungsfrage ebenfalls «Ja». War sie es nicht, dann:

3. Durchführung einer visuellen Analyse des gesamten Prozesses, wie von Borckardt \& Nash (2014) empfohlen. Lagen offensichtliche Auffälligkeiten im Prozess vor, die die ES beeinflussten und vom Trend abwichen, war die Antwort ebenfalls «Ja». Wenn nicht, war die Antwort auf die Forschungsfrage «Nein».

Weitere Erwägungen für die Datenanalyse: Für die Ergänzung fehlender Daten wurde das EM-Verfahren (Expectation-Maximation-Algorithmus) angewendet, eine für Zeitreihen-Beobachtungen gut geeignete Methode, da die Teststärke-Sensitivität sinkt, wenn die Autokorrelation hoch ist (Smith et al., 2012). Meta-Analyse war ein wichtiger Teil unserer Studie, da sie es uns ermöglichte: (1) die Effektstärken (ES) für alle Zielbeschwerden (TC) und das Glass' $\Delta$ zu berechnen, wobei sie mit den ESWerten verglichen wurden, die durch die SMA erhalten wurden, (2) einen aggregierten ES-Wert für jeden der zehn Fälle zu erhalten, und (3) aggregierte ES-Werte für die gesamte Studie zu berechnen und den Platz jedes Falls im Kontext aller Fälle zu bestimmen (Manolov \& Solanas, 2008). Für diesen Zweck berechneten wir die mittleren Standardabweichungen für jede TC mit Glass' $\Delta$ (Glass et al., 1981) wie von Beretvas \& Chung (2008) empfohlen, mittels ungewichteter Durchschnittswerte (siehe Manolov et al., 2014). Die mittlere Standardabweichung hat in einem Einzelfall-Zeitreihendesign bekanntermassen im Gegensatz zum Gruppendesign keine Vergleichswerte und ihre Werte werden durch Spezifität jeder Studie bestimmt.

\section{Ergebnisse}

Unsere Ergebnisse lieferten in dieser Studie Nachweise für die Wirksamkeit der Gestalttherapie. Eine Meta-Analyse aller untersuchten Fälle und ein Beispiel der genaueren Ergebnisse eines ausgewählten Falles werden ebenfalls vorgestellt, um die Antworten zu den drei Forschungsfragen zu ergänzen. In Tabelle 2 sind detaillierte mittlere Werte (M) und der Wert der Standardabweichung (SD) für alle TC dargestellt.

\section{Forschungsfrage I:}

Gibt es im Vergleich vor und nach der Intervention eine Verbesserung, und falls ja, wie gross ist diese?

Wie in Tabelle 3 dargestellt, konnte für fast alle Patientinnen für alle Zielbeschwerden eine therapeutische Veränderung zwischen Baseline und der Nachbeobachtungsphase nachgewiesen werden. Die einzige Ausnahme stellte Zielbeschwerde Nr. 2 (TC2) von Patientin 7 dar: «Ich kann das Mobbing an meinem Arbeitsplatz nicht ertragen.» Diese spezielle TC zeigte eine leichte Verschlechterung $(\mathrm{r}=+0.353, \mathrm{p}=0.4546)$, beginnend mit 3,9 (Baseline-Durchschnitt) und endend mit 5,1 (Nachbeobachtungsdurchschnitt). Wir können dies als ein Problem mit der Definition dieser Zielbeschwerde interpretieren.

Alle drei Indikatoren bestätigten das Vorhandensein von Veränderungen bei den verbleibenden 30 Zielbeschwerden. Die MBLR-Werte bewegten sich zwischen $28 \%$ und $88 \%$; die Pearsons Korrelation der TC mit Phasenvektor wies Werte zwischen -0.585 und -0.996 auf (TC2 von Patientin 6 hat einen r-Wert von -1.00, ist aber ein Sonderfall, indem es in beiden Phasen absolut keine Varianz gab); und die visuelle Analyse zeigte verschiedene Abstufungen der Besserung auf, sowohl sukzessiv (z. B. Patientin 4, TC2) als auch plötzlich (z. B. Patientin 1, TC1). Alle Rohdaten (einschliesslich TC-Werten und Grafiken für jedes TC) sind offen verfügbar, ${ }^{2}$ da wir in diesem Artikel nur ein paar Beispiele der TC-Grafik vorstellen werden.

Von den 30 TC, bei denen eine therapeutische Veränderung nachgewiesen werden konnte, war die Veränderung bei 21 TC gross (MBLR > 65\%; $r<-0.749$; $p<0.05$; plus merkliche Veränderung in der visuellen Analyse). Bei 6 TC war die Veränderung mittlerer Stärke (MBLR > 51\%; $\mathrm{r}<-0.611 ; \mathrm{p}<0.05$; plus signifikante Veränderung in der visuellen Analyse). Bei den verbleibenden 3 TC wurde die Veränderung als gering angesehen (MBLR $>28 \%$; $r<-0.585 ; p<0.05$; plus merkliche Veränderung in der visuellen Analyse).

\section{Forschungsfrage II: Ist die Veränderung klinisch bedeutsam?}

Wie in Tabelle 4 dargestellt, war bei neun der zehn Fälle eine therapeutisch bedeutsame Veränderung zu beobachten, wohingegen die Ergebnisse von Patientin 2 strittig waren. Im Fall von Patientin 2 zeigten zwei der drei Zielbeschwerden eine bedeutsame Veränderung.

Bei Patientin 2 zeigten die Tests auf Level-Änderung und MBLR-Werte eine geringe oder mittlere Veränderung der Zielbeschwerden an, aber die EB-45-Werte sanken zwischen der Baseline- und der Nachbeobachtungsphase nicht. Die Patientin begann laut ihren EB-Werten jedoch im «funktionalen» Bereich und die Hamilton-AnxietyWerte sanken zwischen Baseline und der Nachbeobachtung sieben Monate nach Therapieende von mittelschwer (21) auf leicht (8).

In allen andern neun Fällen zeigten die EB-45-Werte eine signifikante Reduktion über dem Minimum des Reliable Change Index von 17. Ausserdem zeigten alle anderen Patientinnen eine Verbesserung ihrer Anxiety-Werte (von mittelschwer auf leicht oder von schwer auf leicht im Fall von Patientin 7) sowie ihrer BDI-Werte (von mittlere auf mild oder von mild auf klinisch unauffällig bei den Patientinnen 6 und 4). Zum Beispiel wechselte Patientin 3 von der klinischen Population in die Normalpopulation,

2 https://drive.google.com/open?id=0Bwx6E-BamGktVzlnU3NsTmNMUXc 


\begin{tabular}{|c|c|c|c|c|c|c|c|}
\hline \multirow[t]{2}{*}{ Nr. } & TC (Nr. und Text) & \multicolumn{2}{|c|}{ Baseline } & \multicolumn{2}{|c|}{ Therapie } & \multicolumn{2}{|c|}{$\begin{array}{l}\text { Nach- } \\
\text { beob- } \\
\text { achtung }\end{array}$} \\
\hline & & M & SD & M & SD & M & SD \\
\hline \multirow{5}{*}{1} & & \multicolumn{2}{|c|}{$\mathrm{N}=13$} & \multicolumn{2}{|c|}{$\mathrm{N}=155$} & \multicolumn{2}{|c|}{$\mathrm{N}=14$} \\
\hline & 1 Ich kann meine wahren Gefühle nicht zeigen & 5,1 & 0,3 & 4,3 & 1,7 & 1 & 0,0 \\
\hline & $\begin{array}{l}2 \text { In einer Beziehung bin ich unnachgiebig, es ist schwer, } \\
\text { mich emotional darauf einzulassen }\end{array}$ & 4,4 & 1,0 & 4,6 & 1,9 & 1,2 & 0,4 \\
\hline & 3 Distanzierte Beziehung zu Eltern & 4 & 1,1 & 2,7 & 1,3 & 1,1 & 0,4 \\
\hline & 4 Habe meinen Zorn nicht gut im Griff & 4,5 & 1,1 & 1,6 & 1,0 & 1 & 0,0 \\
\hline \multirow{4}{*}{2} & & \multicolumn{2}{|c|}{$\mathrm{N}=17$} & \multicolumn{2}{|c|}{$\mathrm{N}=149$} & \multicolumn{2}{|c|}{$\mathrm{N}=12$} \\
\hline & 1 Angst vor Erfolg & 3,3 & 1,0 & 2,7 & 1,0 & 2,2 & 0,4 \\
\hline & 2 Angststörung & 3,7 & 0,8 & 2,9 & 1,1 & 2,3 & 0,6 \\
\hline & 3 Schlechte Beziehung zur Mutter & 2,1 & 0,9 & 1,4 & 0,6 & 1 & 0,0 \\
\hline \multirow{4}{*}{3} & & \multicolumn{2}{|c|}{$\mathrm{N}=16$} & \multicolumn{2}{|c|}{$\mathrm{N}=120$} & \multicolumn{2}{|c|}{$\mathrm{N}=11$} \\
\hline & 1 Ich bin traurig darüber, dass ich keinen Kontakt zu anderen aufnehme & 3,9 & 0,9 & 2,7 & 1,2 & 1,6 & 0,9 \\
\hline & 2 Ich fühle mich schuldig, wenn ich meine eigenen Anforderungen nicht erfülle & 5,1 & 0,8 & 3,5 & 1,5 & 2,1 & 0,8 \\
\hline & 3 Ich vermeide es, meine Wut zu zeigen & 3 & 1,3 & 2,2 & 1,1 & 1,5 & 0,8 \\
\hline \multirow{4}{*}{4} & & \multicolumn{2}{|c|}{$\mathrm{N}=13$} & \multicolumn{2}{|c|}{$\mathrm{N}=148$} & \multicolumn{2}{|c|}{$\mathrm{N}=14$} \\
\hline & 1 Ich bin für meine Familie unzulänglich & 4,5 & 1,0 & 2,5 & 1,3 & 1 & 0,0 \\
\hline & 2 Ich bin verzweifelt, wenn ich unbeschäftigt bin & 4,8 & 1,5 & 2,4 & 1,4 & 1 & 0,0 \\
\hline & 3 Ich fühle mich regelmässig gequält & 3,8 & 1,1 & 2,2 & 1,5 & 1 & 0,0 \\
\hline \multirow{4}{*}{5} & & \multicolumn{2}{|c|}{$\mathrm{N}=17$} & \multicolumn{2}{|c|}{$\mathrm{N}=238$} & & \\
\hline & $\begin{array}{l}1 \text { Ich kann keine ausreichende Abgrenzung zu meinem Ex herstellen, } \\
\text { sodass er sich nicht verhält, als seien wir noch immer ein Paar }\end{array}$ & 5,9 & 1,3 & 3,9 & 2,0 & 1,6 & 1,2 \\
\hline & $\begin{array}{l}2 \text { Ich kann nicht freundlich mit anderen umgehen, } \\
\text { ich bin immer distanziert oder aggressiv }\end{array}$ & 4,8 & 1,9 & 2,5 & 1,9 & 1,2 & 0,6 \\
\hline & $\begin{array}{l}3 \text { Angststörung: Ich kann nicht akzeptieren was passiert, } \\
\text { meine Brust wird eng und ich habe negative Gedanken }\end{array}$ & 5,9 & 1,6 & 3,7 & 2,1 & 1,6 & 1,3 \\
\hline & & $\mathrm{N}=$ & & $\mathrm{N}=$ & & & \\
\hline 6 & 1 Es fällt mir schwer Dinge zu geniessen & 4,2 & 0,8 & 1,8 & 0,8 & 1 & 0,0 \\
\hline 0 & 2 Ich habe in Bezug auf Kaufen und Essen keine Selbstdisziplin & 7 & 0,0 & 5,5 & 0,6 & 5 & 0,0 \\
\hline & 3 Ich kann meinen Körper nicht akzeptieren & 4,8 & 0,7 & 3,4 & 0,9 & 2 & 0,0 \\
\hline & & $\mathrm{N}=$ & & $\mathrm{N}=$ & & & \\
\hline 7 & 1 Ich fühle mich als Mutter nicht sicher & 4,5 & 1,4 & 3,3 & 1,9 & 1,5 & 0,5 \\
\hline 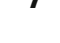 & 2 Ich kann das Mobbing an meinem Arbeitsplatz nicht ertragen & 3,9 & 1,7 & 4,4 & 1,4 & 5,1 & 1,6 \\
\hline & 3 Ich kann mich dem Leben mit meiner gespaltenen Familie kaum stellen & 4 & 1,5 & 3,4 & 1,4 & 1,1 & 0,3 \\
\hline & & $\mathrm{N}=$ & & $\mathrm{N}=$ & & & \\
\hline 8 & 1 Angst davor, die Erwartungen von jemand anderem nicht zu erfüllen & 7,2 & 1,0 & 5 & 2,3 & 2,5 & 1,5 \\
\hline 0 & 2 Angst davor, einen Fehler zu machen & 6,5 & 1,2 & 3,7 & 1,9 & 2 & 1,7 \\
\hline & 3 Lehnt sich selbst ab & 6,1 & 1,8 & 5 & 1,8 & 2,7 & 1,7 \\
\hline & & $\mathrm{N}=$ & & $\mathrm{N}=$ & & & \\
\hline 0 & 1 Ich fühle mich anderen Menschen gegenüber unsicher & 6,9 & 1,0 & 3,7 & 1,6 & 1,5 & 0,6 \\
\hline & 2 Ich habe Angst vor der Geringschätzung anderer Menschen & 7,1 & 1,1 & 3,5 & 1,6 & 1,6 & 0,7 \\
\hline & 3 Ich habe Probleme damit, beim Sex ungehemmt und frei zu sein & 8,2 & 1,1 & 4,2 & 2,1 & 1 & 0,0 \\
\hline & & $\mathrm{N}=$ & & $\mathrm{N}=$ & & & \\
\hline & 1 Ich weiss nicht weiter, ich hinterfrage ständig, was ich tue & 5,7 & 2,0 & 4,2 & 1,9 & 1,9 & 0,5 \\
\hline 10 & 2 Ich habe keine Gefühle, Dinge berühren mich nicht wie früher & 7,5 & 1,3 & 2,1 & 1,3 & 1 & 0,0 \\
\hline & $\begin{array}{l}3 \text { Wenn es nicht gut für mich läuft, bekomme ich Angst und fürchte, } \\
\text { eine Panikattacke zu bekommen }\end{array}$ & 8,6 & 1,0 & 5 & 2,7 & 1,1 & 0,5 \\
\hline
\end{tabular}

Tab. 2: Beschreibung der Zielbeschwerden für alle Fälle 


\begin{tabular}{|c|c|c|c|c|c|c|c|c|}
\hline \multirow[b]{2}{*}{$\begin{array}{l}\text { Patien- } \\
\text { tinnen- } \\
\text { Nr. }\end{array}$} & \multirow[b]{2}{*}{ TC-Nr. } & \multicolumn{4}{|c|}{$\begin{array}{l}\text { Gibt es im Vergleich vor und nach der Intervention } \\
\text { eine Veränderung, und falls JA, wie gross ist diese? }\end{array}$} & \multicolumn{3}{|c|}{$\begin{array}{l}\text { Kann diese Änderung der The- } \\
\text { rapie zugeschrieben werden? }\end{array}$} \\
\hline & & $\begin{array}{l}r \text { und } p \text { in } \\
\text { SMA }\end{array}$ & $\begin{array}{l}\text { MBLR } \\
(\%)\end{array}$ & Visuelle Analyse & $\begin{array}{l}\text { Ja/Nein? } \\
\text { Grösse }\end{array}$ & $\begin{array}{l}\text { Gesamt- } \\
\text { trend } \\
\left(\mathrm{R}^{2}\right)^{*}\end{array}$ & $\begin{array}{l}x y / z \\
\text { und } p \text { in } \\
\text { SMA }\end{array}$ & $\begin{array}{l}\text { Ja/ } \\
\text { Nein? }\end{array}$ \\
\hline \multirow{4}{*}{1} & 1 & $\begin{array}{l}-0.996 \\
0.0001\end{array}$ & 80 & $\begin{array}{l}\text { Grosse Verbesserung in } \\
\text { den letzten Wochen der Therapie }\end{array}$ & Ja, gross & Nein & & Ja \\
\hline & 2 & $\begin{array}{l}-0.914 \\
0.0002\end{array}$ & 72 & $\begin{array}{l}\text { Grosse Verbesserung in } \\
\text { den letzten Wochen der Therapie }\end{array}$ & Ja, gross & Nein & & Ja \\
\hline & 3 & $\begin{array}{l}-0.882 \\
0.0001\end{array}$ & 71 & $\begin{array}{l}\text { Stabile Verbesserung } \\
\text { während der Therapiephase }\end{array}$ & Ja, gross & 0.536 & $\begin{array}{l}-0.733 \\
0.0206\end{array}$ & Ja \\
\hline & 4 & $\begin{array}{l}-0.920 \\
0.0012\end{array}$ & 78 & $\begin{array}{l}\text { Schnelle Verbesserung während } \\
\text { Baseline bis zum ersten Teil der } \\
\text { Therapiephase }\end{array}$ & Ja, gross & 0.617 & $\begin{array}{r}-0.673 \\
0.163\end{array}$ & $\begin{array}{r}\text { Frag- } \\
\text { lich }\end{array}$ \\
\hline \multirow{3}{*}{2} & 1 & $\begin{array}{l}-0.585 \\
0.0216\end{array}$ & 34 & $\begin{array}{l}\text { Langsame Verbesserung } \\
\text { während der Therapie }\end{array}$ & Ja, klein & Nein & & Ja \\
\hline & 2 & $\begin{array}{l}-0.697 \\
0.0036\end{array}$ & 39 & $\begin{array}{l}\text { Langsame Verbesserung } \\
\text { während der Therapie }\end{array}$ & Ja, klein & Nein & & Ja \\
\hline & 3 & $\begin{array}{l}-0.624 \\
0.0244\end{array}$ & 52 & $\begin{array}{l}\text { Langsame Verbesserung } \\
\text { während Baseline und } \\
\text { zu Beginn der Therapie }\end{array}$ & Ja, mittel & Nein & & Ja \\
\hline \multirow{3}{*}{3} & 1 & $\begin{array}{l}-0.765 \\
0.0022\end{array}$ & 55 & $\begin{array}{l}\text { Sukzessive Verbesserung } \\
\text { während der Therapie }\end{array}$ & Ja, mittel & 0.454 & $\begin{array}{r}-0.67 \\
0.0148\end{array}$ & Ja \\
\hline & 2 & $\begin{array}{l}-0.862 \\
0.002\end{array}$ & 51 & $\begin{array}{l}\text { Sukzessive Verbesserung } \\
\text { während der Therapie }\end{array}$ & Ja, mittel & 0.563 & $\begin{array}{l}-0.805 \\
0.0076\end{array}$ & Ja \\
\hline & 3 & $\begin{array}{l}-0.611 \\
0.0148\end{array}$ & 52 & $\begin{array}{l}\text { Sukzessive Verbesserung } \\
\text { während der Therapie }\end{array}$ & Ja, mittel & Nein & & Ja \\
\hline \multirow{3}{*}{4} & 1 & $\begin{array}{l}-0.939 \\
0.0001\end{array}$ & 78 & $\begin{array}{l}\text { Sukzessive, aber unbeständige } \\
\text { Verbesserung während der Therapie }\end{array}$ & Ja, gross & 0.574 & $\begin{array}{l}-0.771 \\
0.0864\end{array}$ & Ja \\
\hline & 2 & $\begin{array}{l}-0.892 \\
0.0002\end{array}$ & 79 & $\begin{array}{l}\text { Sukzessive Verbesserung } \\
\text { während der Therapie }\end{array}$ & Ja, gross & 0.653 & $\begin{array}{l}-0.686 \\
0.0438\end{array}$ & $\mathrm{Ja}$ \\
\hline & 3 & $\begin{array}{l}-0.881 \\
0.0012\end{array}$ & 74 & $\begin{array}{l}\text { Sukzessive und sehr ungleichmässige } \\
\text { Verbesserung während } \\
\text { der ersten Hälfte der Therapie }\end{array}$ & Ja, gross & 0.603 & $\begin{array}{l}-0.572 \\
0.1614\end{array}$ & $\begin{array}{r}\text { Frag- } \\
\text { lich }\end{array}$ \\
\hline \multirow{3}{*}{5} & 1 & $\begin{array}{l}-0.871 \\
0.0001\end{array}$ & 73 & Sehr ungleichmässige Verbesserung & Ja, gross & 0.448 & $\begin{array}{l}-0.775 \\
0.0062\end{array}$ & Ja \\
\hline & 2 & $\begin{array}{l}-0.820 \\
0.0002\end{array}$ & 76 & Sehr ungleichmässige Verbesserung & Ja, gross & 0.375 & $\begin{array}{l}-0.722 \\
0.0084\end{array}$ & Ja \\
\hline & 3 & $\begin{array}{l}-0.839 \\
0.001\end{array}$ & 73 & Sehr ungleichmässige Verbesserung & Ja, gross & 0.394 & $\begin{array}{l}-0.749 \\
0.0052\end{array}$ & Ja \\
\hline
\end{tabular}




\begin{tabular}{|c|c|c|c|c|c|c|c|c|}
\hline \multirow[b]{2}{*}{$\begin{array}{l}\text { Patien- } \\
\text { tinnen- } \\
\text { Nr. }\end{array}$} & \multirow[b]{2}{*}{ TC-Nr. } & \multicolumn{4}{|c|}{$\begin{array}{l}\text { Gibt es im Vergleich vor und nach der Intervention } \\
\text { eine Veränderung, und falls JA, wie gross ist diese? }\end{array}$} & \multicolumn{3}{|c|}{$\begin{array}{l}\text { Kann diese Änderung der The- } \\
\text { rapie zugeschrieben werden? }\end{array}$} \\
\hline & & $\begin{array}{l}r \text { und } p \text { in } \\
\text { SMA }\end{array}$ & $\begin{array}{c}\text { MBLR } \\
(\%)\end{array}$ & Visuelle Analyse & $\begin{array}{l}\text { Ja/Nein? } \\
\text { Grösse }\end{array}$ & $\begin{array}{l}\text { Gesamt- } \\
\text { trend } \\
\left(\mathrm{R}^{2}\right)^{*}\end{array}$ & $\begin{array}{l}x y / z \\
\text { und } p \text { in } \\
\text { SMA }\end{array}$ & $\begin{array}{l}\text { Ja/ } \\
\text { Nein? }\end{array}$ \\
\hline \multirow{3}{*}{6} & 1 & $\begin{array}{l}-0.931 \\
0.0002\end{array}$ & 76 & Sukzessive Verbesserung & Ja, gross & 0.726 & $\begin{array}{l}-0.836 \\
0.0152\end{array}$ & Ja \\
\hline & 2 & $\begin{array}{l}-1.000 \\
0.0001\end{array}$ & 28 & Geringe sukzessive Verbesserung & Ja, klein & 0.784 & $\begin{array}{r}-1 \\
0.0001\end{array}$ & Ja \\
\hline & 3 & $\begin{array}{l}-0.926 \\
0.0001\end{array}$ & 58 & $\begin{array}{l}\text { Sukzessive, aber unregelmässige } \\
\text { Verbesserung }\end{array}$ & Ja, mittel & 0.537 & $\begin{array}{r}-0.844 \\
0.004\end{array}$ & $\mathrm{Ja}$ \\
\hline \multirow{3}{*}{7} & 1 & $\begin{array}{l}-0.828 \\
0.0086\end{array}$ & 67 & $\begin{array}{l}\text { Starke Verbesserung } \\
\text { in der ersten Hälfte der Therapie }\end{array}$ & Ja, gross & Nein & & $\mathrm{Ja}$ \\
\hline & 2 & $\begin{array}{l}+0.353 \\
0.4546\end{array}$ & -32 & Keine Verbesserung, unregelmässig & Nein & Nein & & - \\
\hline & 3 & $\begin{array}{l}-0.812 \\
0.0162\end{array}$ & 73 & $\begin{array}{l}\text { Sehr ungleichmässig, Verbesserung } \\
\text { am Ende der Therapiephase }\end{array}$ & Ja, gross & 0.545 & $\begin{array}{l}-0.332 \\
0.5012\end{array}$ & $\mathrm{Ja}$ \\
\hline \multirow{3}{*}{8} & 1 & $\begin{array}{l}-0.804 \\
0.0008\end{array}$ & 65 & $\begin{array}{l}\text { Unregelmässig, aber grosse } \\
\text { Verbesserung während der Therapie }\end{array}$ & Ja, gross & 0.515 & $\begin{array}{l}-0.569 \\
0.1048\end{array}$ & $\mathrm{Ja}$ \\
\hline & 2 & $\begin{array}{l}-0.749 \\
0.0006\end{array}$ & 68 & $\begin{array}{l}\text { Grosse Verbesserung } \\
\text { im ersten Drittel der Therapie }\end{array}$ & Ja, gross & 0.578 & $\begin{array}{l}-0.476 \\
0.1590\end{array}$ & Ja \\
\hline & 3 & $\begin{array}{l}-0.635 \\
0.0082\end{array}$ & 56 & $\begin{array}{l}\text { Unregelmässig, } \\
\text { aber grosse Verbesserung während } \\
\text { der Therapie und Nachbeobachtung }\end{array}$ & Ja, mittel & 0,37 & $\begin{array}{l}-0.282 \\
0.4062\end{array}$ & Ja \\
\hline \multirow{3}{*}{9} & 1 & $\begin{array}{l}-0.958 \\
0.0001\end{array}$ & 78 & $\begin{array}{l}\text { Sukzessive und grosse Verbesse- } \\
\text { rung im ersten Drittel der Therapie }\end{array}$ & $\mathrm{Ja}$, gross & 0.436 & $\begin{array}{l}-0.894 \\
0.0018\end{array}$ & Ja \\
\hline & 2 & $\begin{array}{l}-0.951 \\
0.0002\end{array}$ & 78 & $\begin{array}{l}\text { Sukzessive und grosse Verbesse- } \\
\text { rung im ersten Drittel der Therapie }\end{array}$ & $\mathrm{Ja}$, gross & 0.428 & $\begin{array}{l}-0.866 \\
0.0046\end{array}$ & $\mathrm{Ja}$ \\
\hline & 3 & $\begin{array}{l}-0.981 \\
0.0001\end{array}$ & 88 & $\begin{array}{l}\text { Sukzessive und grosse Verbesse- } \\
\text { rung im ersten Drittel der Therapie }\end{array}$ & Ja, gross & 0.713 & $\begin{array}{l}-0.930 \\
0.0014\end{array}$ & $\mathrm{Ja}$ \\
\hline \multirow{3}{*}{10} & 1 & $\begin{array}{l}-0.854 \\
0.0008\end{array}$ & 71 & $\begin{array}{l}\text { Sehr unregelmässig, aber Besserung } \\
\text { während der Nachbeobachtung }\end{array}$ & Ja, gross & Nein & & Ja \\
\hline & 2 & $\begin{array}{l}-0.973 \\
0.0001\end{array}$ & 87 & $\begin{array}{l}\text { Grosse Verbesserung } \\
\text { zu Beginn der Therapie }\end{array}$ & Ja, gross & 0.33 & $\begin{array}{l}-0.923 \\
0.0001\end{array}$ & $\mathrm{Ja}$ \\
\hline & 3 & $\begin{array}{l}-0.982 \\
0.0001\end{array}$ & 85 & $\begin{array}{l}\text { Sehr unregelmässig, aber Besserung } \\
\text { während der Nachbeobachtung }\end{array}$ & $\mathrm{Ja}$, gross & 0.757 & $\begin{array}{l}-0.963 \\
0.0001\end{array}$ & $\mathrm{Ja}$ \\
\hline
\end{tabular}

Tab. 3: Ergebnisse der Forschungsfragen I und III 


\begin{tabular}{|c|c|c|c|c|c|c|}
\hline $\begin{array}{c}\text { Patien- } \\
\text { tinnen- } \\
\text { Nr. }\end{array}$ & Diagnose & Messung & Sitzung o & $\begin{array}{l}\text { Letzte } \\
\text { Therapie- } \\
\text { sitzung }\end{array}$ & $\begin{array}{l}\text { Nachbe- } \\
\text { obach- } \\
\text { tungs- } \\
\text { sitzung }\end{array}$ & $\begin{array}{l}\text { Schluss- } \\
\text { folger- } \\
\text { ung }\end{array}$ \\
\hline \multirow{2}{*}{1} & \multirow{2}{*}{ Angststörung } & Hamilton-Test & 13 & 3 & 3 & \multirow{2}{*}{ Ja } \\
\hline & & EB-45 & 50 & $9^{*}$ & $14^{*}$ & \\
\hline \multirow{2}{*}{2} & \multirow{2}{*}{ Generalisierte Angststörung } & Hamilton-Test & 21 & 20 & 8 & \multirow{2}{*}{ Fraglich } \\
\hline & & EB-45 & 54 & 52 & 57 & \\
\hline \multirow{3}{*}{3} & \multirow{3}{*}{ Angststörung } & Hamilton-Test & 17 & 10 & 12 & \multirow{3}{*}{$\mathrm{Ja}$} \\
\hline & & EB-45 & 67 & $39^{*}$ & $29^{*}$ & \\
\hline & & BDI & 13 & 3 & 7 & \\
\hline \multirow{3}{*}{4} & \multirow{3}{*}{ Alkoholmissbrauch, Agoraphobie, Depression } & Hamilton-Test & 19 & 6 & 4 & \multirow{3}{*}{$\mathrm{Ja}$} \\
\hline & & EB-45 & 62 & $21^{*}$ & $34^{*}$ & \\
\hline & & BDI & 11 & 3 & 2 & \\
\hline \multirow{3}{*}{5} & \multirow{3}{*}{$\begin{array}{l}\text { Panik - Agoraphobie, } \\
\text { generalisierte Angststörung }\end{array}$} & Hamilton-Test & 29 & 9 & 9 & \multirow{3}{*}{$\mathrm{Ja}$} \\
\hline & & EB-45 & 90 & $59^{*}$ & $44^{*}$ & \\
\hline & & BDI & 19 & 7 & 4 & \\
\hline \multirow{3}{*}{6} & \multirow{3}{*}{ Angst und depressive Störung, gemischt } & Hamilton-Test & 17 & 6 & 5 & \multirow{3}{*}{$\mathrm{Ja}$} \\
\hline & & EB-45 & 49 & 40 & $27^{*}$ & \\
\hline & & BDI & 11 & 7 & 5 & \\
\hline \multirow{3}{*}{7} & \multirow{3}{*}{ Angst und depressive Störung, gemischt } & Hamilton-Test & 39 & 12 & 8 & \multirow{3}{*}{$\begin{array}{l}\text { Ja (für zwei } \\
\text { der drei TC) }\end{array}$} \\
\hline & & EB-45 & 96 & $76^{*}$ & $70^{*}$ & \\
\hline & & BDI & 21 & 5 & 7 & \\
\hline \multirow{3}{*}{8} & \multirow{3}{*}{ Angststörung } & Hamilton-Test & 25 & 10 & 8 & \multirow{3}{*}{$\mathrm{Ja}$} \\
\hline & & EB-45 & 99 & $49^{*}$ & 42 & \\
\hline & & BDI & 21 & 7 & 5 & \\
\hline \multirow{3}{*}{9} & \multirow{3}{*}{ Anpassungsstörung mit Angstsymptomen } & Hamilton-Test & 23 & 10 & 13 & \multirow{3}{*}{$\mathrm{Ja}$} \\
\hline & & EB-45 & 77 & $41^{*}$ & 40 & \\
\hline & & BDI & 23 & 7 & 5 & \\
\hline \multirow{3}{*}{10} & \multirow{3}{*}{ Panik ohne Agoraphobie } & Hamilton-Test & 24 & 13 & 10 & \multirow{3}{*}{$\mathrm{Ja}$} \\
\hline & & EB-45 & 91 & $59^{*}$ & $39^{*}$ & \\
\hline & & BDI & 21 & 4 & 3 & \\
\hline
\end{tabular}

Tab. 4: Ergebnisse der Forschungsfrage II

wies einen klinisch signifikanten Veränderungsindex auf und ihre Angststörung konnte von «mittelschwer» auf «keine Angststörung» heruntergestuft werden.

Zusammenfassend gesagt betrug der durchschnittliche Hamilton-Wert zu Beginn in Sitzung 0 22,7 und verbesserte sich auf 9,9 in der Abschlusssitzung bzw. 8,0 in der Nachbeobachtungssitzung nach sechs Monaten. Die durchschnittlichen EB-45-Werte lagen zu Beginn bei 73,5 und verbesserten sich auf 46 in der Abschlusssitzung und auf 46,3 bei der Nachbeobachtung. Auch die BDIWerte verbesserten sich von 17 in Sitzung 0 auf 5,6 in der Abschlusssitzung und 5,0 in der Nachbeobachtung. Insgesamt konnte mit den Ergebnissen gezeigt werden, dass in neun der zehn Fälle die therapeutische Verän- derung definitiv klinisch bedeutsam war und über die Zeit aufrechterhalten werden konnte, wohingegen es im verbleibenden Fall gemischte Indikatoren gab.

\section{Forschungsfrage III: Kann die Verbesserung dem Therapieprozess zugeschrieben werden?}

Der in Tabelle 3 dargestellte dreischrittige Prozess wurde durchgeführt, um zu erforschen, ob die Veränderungen einem allgemeinen Abwärtstrend der Messungen zugeschrieben werden konnten, der keinen Therapiebezug hatte. Nach der Regressionsanalyse erkannten wir, dass neun TC keinen statistisch signifikanten Trend aufwiesen, 
daher berechneten wir ihre partielle Korrelation nicht. Wir erkannten, dass die Verbesserung bei 15 TC der verbleibenden 22 Zielbeschwerden der Therapie zugeschrieben werden konnte, wenn der Einfluss des bestehenden Trends kontrolliert wurde. Die r-Werte der partiellen Korrelation lagen dabei zwischen -0.67 und -1 und alle $\mathrm{p}$-Werte unter 0.05 . In den verbleibenden sieben TC war eine visuelle Analyse notwendig.

Bei fünf Zielbeschwerden zeigte die visuelle Analyse ausgeprägte kurzzeitige Phasen, in denen die TC-Werte stark anstiegen und dadurch die Werte des partiellen Korrelationskoeffizienten verzerrten, aber dies stand in keinem Zusammenhang mit dem Trend. Daher konnten die Veränderungen hier als zur Therapie zugehörig angesehen und nicht dem Einfluss des Trends zugeschrieben werden. Bei den verbleibenden beiden TC waren die Ergebnisse strittig, da die visuelle Analyse einen deutlichen kurzfristigen Trend zeigte, der in der Mitte der Therapiephase endete (z.B. TC3 von Patientin 4 wie nachfolgend im Detail besprochen). Dies könnte von einer für diese Zielbeschwerde im Vergleich zu den restlichen TC erfolgreicheren Therapie verursacht worden sein oder durch eine schnelle natürliche Remission. Für eine endgültige Antwort auf die dritte Forschungsfrage wäre deshalb eine qualitative Analyse des Therapieprozesses erforderlich.

\section{Metaanalyse}

Abbildung 1 zeigt, dass die Fälle 1, 4, 8 ES-Werte haben, die nahe am ES-Wert der gesamten Studie liegen, der 3,2 beträgt. Die Fälle 2, 9 und 10 waren weit davon entfernt und bedürfen daher einer detaillierten qualitativen Analyse.

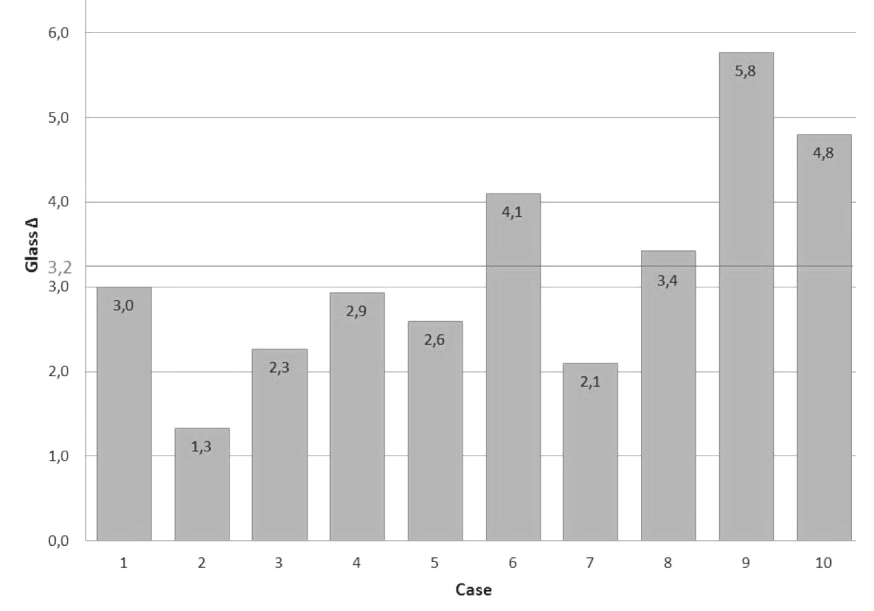

Abb. 1: Metaanalyse aller zehn Fälle

\section{Detaillierte Analyse von Fall-Nr. 4: «Clara»}

Ein Beispiel für die detaillierteren Ergebnisse, die mit dieser Methodologie gewonnen werden können, ist in Fall Nr. 4 dargestellt, der ausgewählt wurde, da seine TC sich hauptsächlich auf Angststörungen beziehen und da der Glass' $\Delta$-Wert von 2,9 nahe am Mittel der gesamten Studie liegt.

Clara war eine 26-jährige Frau, Kinesiologin, ledig, die mit ihrer Mutter und ihren beiden Brüdern zusammenwohnte. Der Therapeut war 33 Jahre alt, in seinem dritten Jahr der Gestalt-Ausbildung und hatte sechs Jahre Erfahrung als Psychotherapeut. Das vom Therapeuten durchgeführte psychiatrische MINI-Interview zeigte eine klinische Diagnose von Agoraphobie, rezidivierender depressiver Störung und Alkoholmissbrauch. Sie zeigte ausserdem eine leichte Depression und eine mittelschwere Angststörung (BDI-1=11; Hamilton=19). Der EB-45 ordnete sie in den funktionalen Bereich ein, sodass sie trotz ihrer Diagnose recht gut funktionieren konnte.

Die visuelle Analyse zeigt, dass die Durchschnittswerte aller TC (gestrichelten Linien in den Grafen in den Abbildungen 2, 3, 4) sich über die drei Phasen deutlich verbessert haben. Dies entspricht der erreichten hohen ES in der Form eines Pearson-Korrelationskoeffizienten der durch eine SMA für alle drei TC errechnet wurde: $\mathrm{r}$ von -0.881 bis -0.939 . Dies weist auf eine enge Beziehung zwischen der Abnahme der TC-Werte hin und darauf, ob sie vor oder nach der Therapie gemessen wurden. Zusätzlich haben hohe Werte eine ES in der Form einer MBLR von $74 \%$ auf $79 \%$, was im Vergleich zu dem vor der Therapie gemessenen Wert auf eine Reduktion des Levels der Beschwerden der Patientin von $75 \%$ hinweist.

Die visuelle Analyse zeigte auch, dass die Abweichung in der Mitte der Therapie für TC1 stark anstieg und in der ersten Hälfte der Therapiephase für TC2 und für alle TC nach der 12-13 Sitzung minimal wird. Auch in allen drei Grafiken ist ein Abwärtstrend zu erkennen, der durch die Werte des R2-Koeffizienten für die entsprechende Regression bestätigt wird: von 0.574 auf 0.653 .

Die Grafik für TC3 zeigt, dass sich die Messungen von der Anfangsphase bis in die folgenden Phasen ändern und nah genug an diesem Trend liegen, wodurch sie ein Beispiel für die Notwendigkeit einer Antwort auf die dritte Forschungsfrage darstellen. Da der partielle Korrelationskoeffizient in der SMA nicht signifikant ( $\mathrm{p}=0.1614)$ war, gibt es einen Grund für die Aussage, dass die Reduktion der TC auf den Einfluss des Trends zurückzuführen ist. Die visuelle Analyse zeigt jedoch ein ähnliches Muster starker Variationen während der Baseline und dem ersten Teil der Therapie, und eine deutliche Verbesserung nach Sitzung 7. Diese Verbesserung stabilisierte sich nach Sitzung 12 und setzte sich in der Nachbeobachtungssitzung neun Monate später fort, als Clara ihre TC3 für die letzte Woche mit einem Durchschnittswert von 1 bewertete. Diese visuelle Analyse lässt darauf schliessen, dass die Veränderungen nicht Folge der natürlichen Remission der Patientin waren, aber bis zu einer detaillierteren qualitativen Analyse bleibt fraglich, ob die Veränderungen der Therapie zugeschrieben werden können.

Der Nachweis von Claras Besserung während der Therapie wird nicht nur durch die Dynamik ihrer 


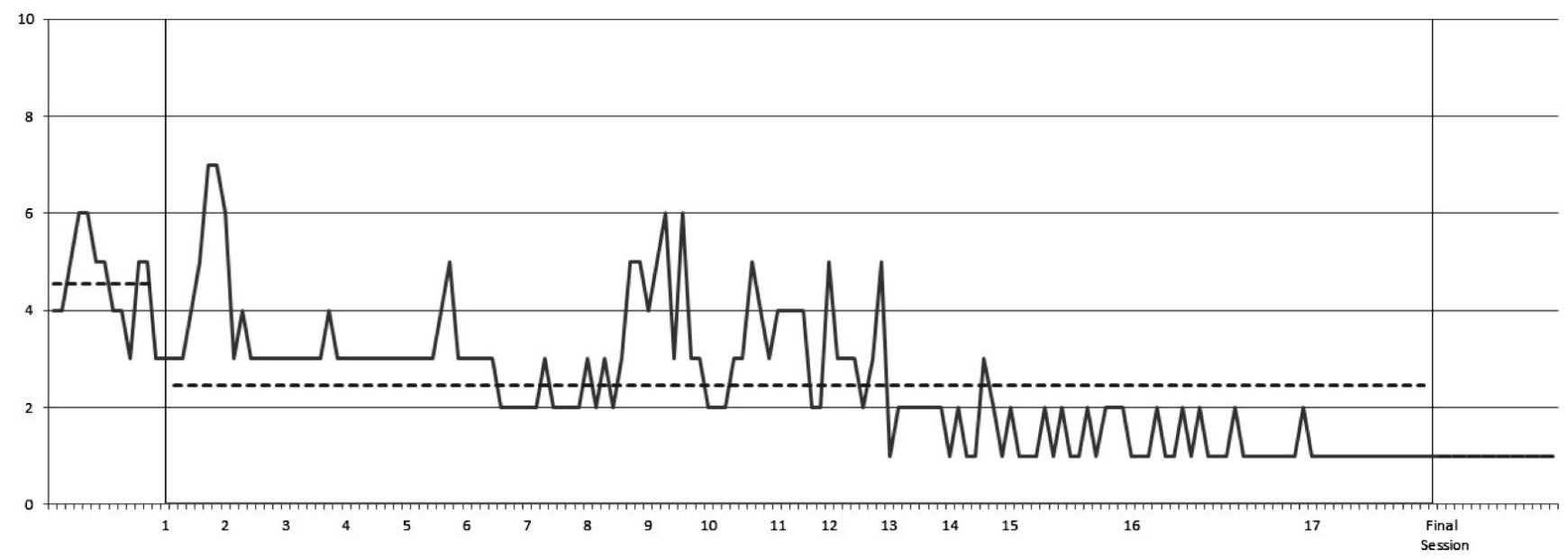

Abb. 2: Zielbeschwerde Nr. 1 während aller drei Phasen («Ich bin für meine Familie unzulänglich.»)

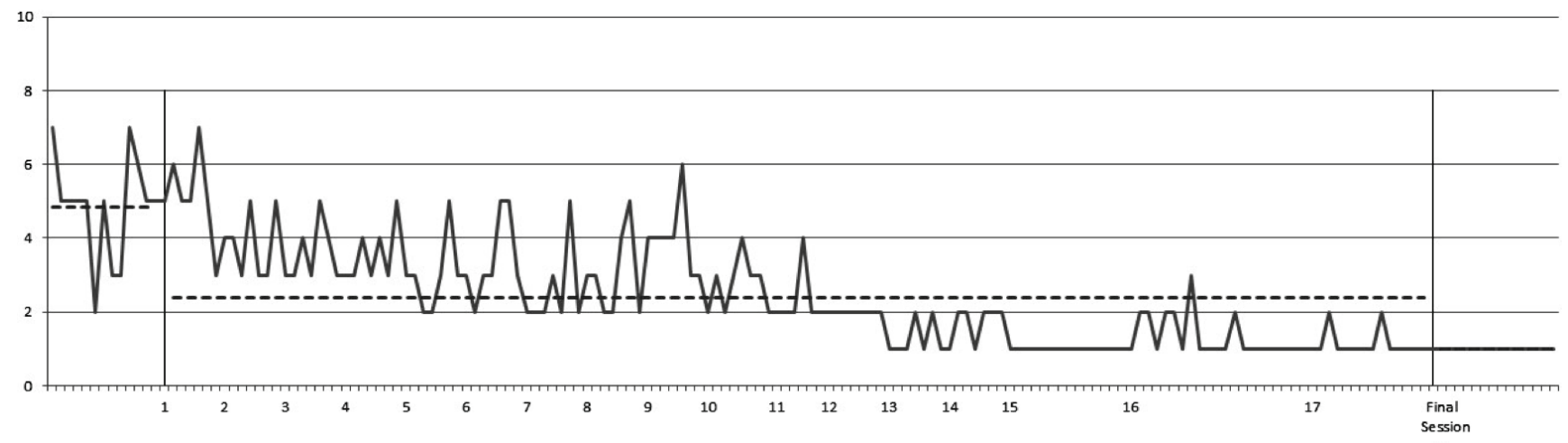

Abb. 3: Zielbeschwerde Nr. 2 während aller drei Phasen («Ich bin verzweifelt, wenn ich unbeschäftigt bin.»)

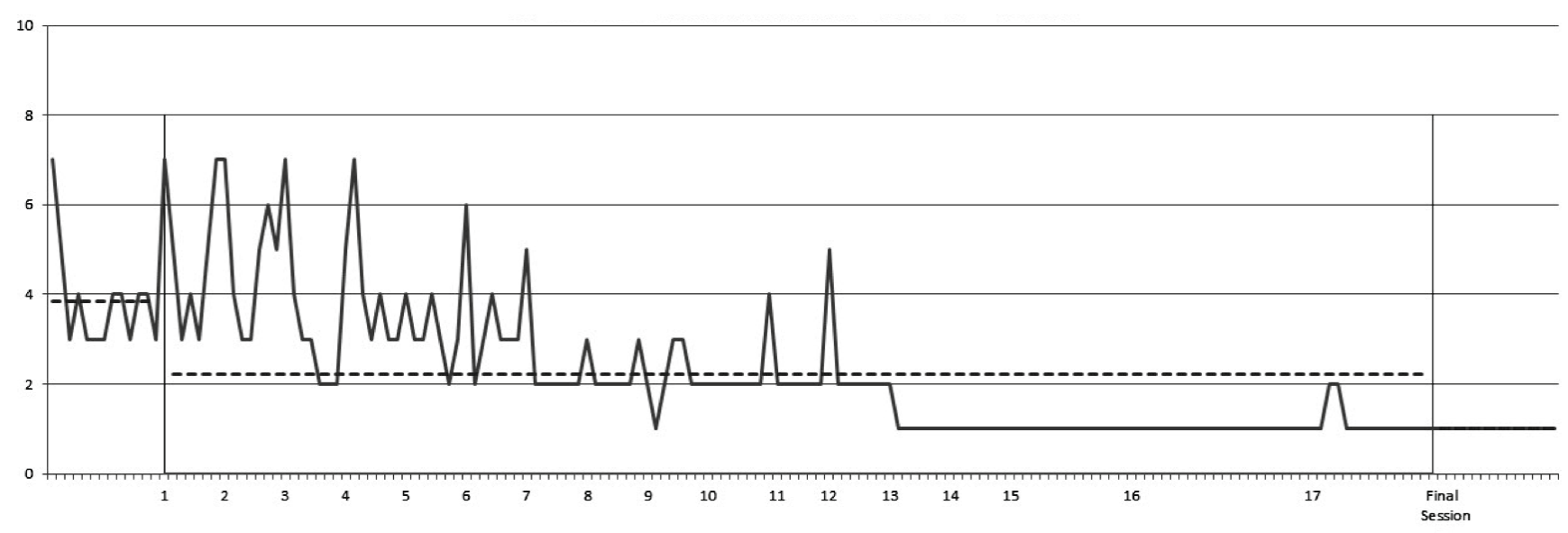

Abb. 4: Zielbeschwerde Nr. 3 während aller drei Phasen («Ich fühle mich regelmässig gequält.») 
Zielbeschwerden erbracht, sondern auch durch die Normalisierung der Indikatoren für Angststörung und Depression (Hamilton $=6$ in der Abschlusssitzung und 4 in der Nachbeobachtungssitzung, BDI = jeweils 3 bzw. 2).

In diesem Fall wurden EB-45-Daten nicht nur zu Beginn und zum Abschluss der Studie erfasst, sondern auch bei jeder Sitzung. In Abbildung 5 ist die Entwicklung von Claras Leiden allgemein abgebildet. EB-45 stieg nach der ersten Sitzung steil an (von 62 in Sitzung 0 auf 102 vor Sitzung 2) und sank dann auf einen Wert, der unter dem Ausgangswert lag (50 vor Sitzung 4). Danach sanken die Leidenswerte nach Sitzung 13 und bis Sitzung 17 sukzessiv und gleichmässig von 48 vor Sitzung 13 auf 20 vor Sitzung 17.

Zusammengenommen zeigt die Fluktuation dieser Werte Wendepunkte während der Therapiephase sowie wichtige psychotherapeutische Momente an, die in einer zukünftigen qualitativen Analyse untersucht werden, die darauf ausgerichtet sein wird, den Veränderungsmechanismus zu verstehen, der die symptomatische Besserung erklärt.

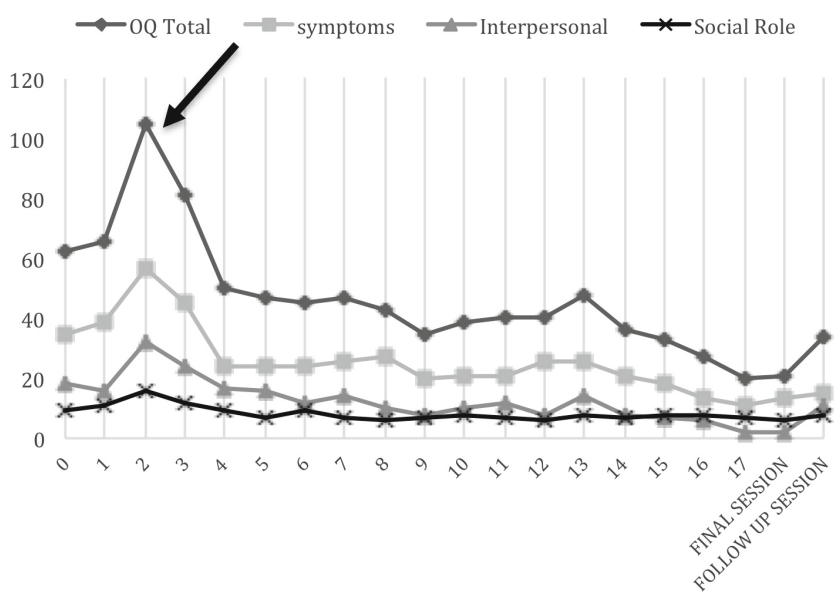

Abb. 5: EB-45-Werte während allen drei Phasen (EB-Gesamt; Symptome; zwischenmenschlich; soziale Integration; Abschlusssitzung; Nachbeobachtungssitzung)

\section{Diskussion}

Ergebnisse der Studie: Die Wirksamkeit der Gestalttherapie wurde in dieser Studie auf mehrere Arten nachgewiesen: Wie in Tabelle 5 abgebildet, konnte bei fast allen TC eine Veränderung zwischen vor und nach der Intervention beobachtet werden, in fast allen Fällen gab es deutliche Indikatoren, dass die Veränderung klinisch bedeutsam war und bei fast allen TC konnte die Veränderung der Therapie zugeschrieben werden. Die zuverlässigen, statistisch signifikanten Ergebnisse unserer Studie lassen darauf schliessen, dass Gestalttherapie (GT) eine praktikable Alternative für andere wirksame Ansätze sein kann und widerlegt so Ergebnisse über die relative Unwirksamkeit von humanistisch-experienziellen (HE) Therapien innerhalb dieser Population (Angus et al., 2015; Lambert, 2013).

\begin{tabular}{|c|c|c|c|}
\hline $\begin{array}{l}\text { Pati- } \\
\text { entin }\end{array}$ & $\begin{array}{c}\text { Veränderung } \\
\text { vorher/nachher? }\end{array}$ & $\begin{array}{l}\text { Klinisch be- } \\
\text { deutsam? }\end{array}$ & $\begin{array}{l}\text { Der Therapie } \\
\text { zuzuschrei- } \\
\text { ben? }\end{array}$ \\
\hline 1 & Ja, gross & $\mathrm{Ja}$ & $\begin{array}{l}\mathrm{Ja}(\mathrm{TC} 1,2,3)+ \\
\text { Fraglich (TC 4) }\end{array}$ \\
\hline 2 & Ja, klein & Fraglich & $\mathrm{Ja}$ \\
\hline 3 & Ja, mittel & $\mathrm{Ja}$ & $\mathrm{Ja}$ \\
\hline 4 & Ja, gross & Ja & $\begin{array}{l}\mathrm{Ja}(\mathrm{TC} 2,3)+ \\
\text { Fraglich (TC 1) }\end{array}$ \\
\hline 5 & Ja, gross & $\mathrm{Ja}$ & $\mathrm{Ja}$ \\
\hline 6 & Ja, mittel & Ja & $\mathrm{Ja}$ \\
\hline 7 & $\begin{array}{l}\text { Ja, gross }(\mathrm{TC} 1,3)+ \\
\text { Keine Verbesserung } \\
(\mathrm{TC} 2)\end{array}$ & $\mathrm{Ja}$ & $\begin{array}{l}\mathrm{Ja}(\mathrm{TC} 2,3)+ \\
\text { Fraglich (TC } 1)\end{array}$ \\
\hline 8 & Ja, gross & $\mathrm{Ja}$ & $\mathrm{Ja}$ \\
\hline 9 & Ja, gross & Ja & Ja \\
\hline 10 & Ja, gross & Ja & $\mathrm{Ja}$ \\
\hline
\end{tabular}

Tab. 5: Zusammenfassung der Ergebnisse

Es ist nicht einfach, unsere Ergebnisse innerhalb der HE-Therapien zu interpretieren, da diese Ansätze alles andere als homogen sind. In der Theorie verfügt der Klientenzentrierte Ansatz (PCA), ein traditionell verbreiteter Ansatz in dieser Gruppe, nicht über die aktiv-direktive Komponente der Gestalttherapie. In der GT werden KlientInnen aktiv ermutigt, sich ihren Ängsten zu stellen, und dabei unterstützt, in ihrer Angststörung ihre ungewollten emotionalen Schemata zu entdecken. Ausserdem werden Rollenspiele angewendet, um die Bedürfnisse der KlientInnen zu erkennen sowie ihre Copingstrategien und Lebenskompetenzen zu verbessern. Sich ungewollten und gefürchteten internen Stimuli zu stellen, fördert emotional korrigierende Erfahrungen. KlientInnen lernen in der GT, dass sie mit den gefürchteten Stimuli umgehen und sie überleben können. Ausserdem wird Vermeidungsreaktionen vorgebeugt. Zusammenfassend lässt sich sagen, dass GT aktive Elemente integriert, die in PCA und einigen anderen HE-Modalitäten fehlen. $\mathrm{Zu}$ diesen Elementen zählen die Angst-Exposition, die Prävention von Vermeidungsverhalten und Schulung der Kompetenzen wie auch in der KVT.

Andererseits teilt die Emotionsfokussierte Therapie (EFT), der am besten durch Forschung gestützte Ansatz der HE-Therapien, die aktiv-direktive Komponente mit GT und wurde auf der Grundlage von GT-Interventionen entwickelt (Greenberg, 1983). Im Gegensatz zu EFT legt GT den Schwerpunkt auf die Arbeit mit der Dynamik der therapeutischen Beziehung im Hier und Jetzt. Die aktuelle GT scheint Elemente von Rogers PCA (humanistische Werte) und EFT (aktive transformationale Interventionen) zu enthalten und fügt insbesondere das dialogische Treffen im Hier und Jetzt sowie holistische Elemente (einschliesslich Körperarbeit) hinzu. Zusammenfassend lässt sich sagen, dass ähnliche Veränderungsprozesse wie 
in der Emotional Processing Theory, dem Modell des inhibitorischen Lernens und der akzeptanzbasierten Therapie vorgeschlagen, zur Erklärung des Veränderungsprozesses in der GT genutzt werden können (Foa \& Kozak, 1986; Craske et al., 2014). Alle diese Modelle treffen im Konzept der «korrigierenden emotionalen Erfahrung» und des "Gedächtnisrekonsolidierungsprozesses» aufeinander, in denen Exposition und insbesondere emotionale Aktivierung von implizit Gelerntem ein wichtiger Veränderungsmechanismus ist (Ecker et al., 2012). Weitere Studien sind notwendig, um die Rolle und den therapeutischen Nutzen von Angst-Exposition zu verstehen, vor allem die Unterschiede ihres Verständnisses in der KVT und humanistischen Theorien. Dies wird Klarheit über die Spezifität der GT und ihre spezifischen Indikationen für Gruppen von PatientInnen schaffen, die davon profitieren könnten.

Obwohl sich unsere Ergebnisse hauptsächlich auf den Vergleich der Baseline- und der Nachbeobachtungsphase beziehen, haben wir die Dynamik der TC über den Verlauf der drei Studienphasen hinweg berücksichtigt. Die virtuelle Analyse nahezu aller TC ergab eine konsistente Verbesserung im Verlauf der Therapie: in manchen Fällen kontinuierlich $(2,3,6)$, in manchen Fällen unregelmässig $(4,5,7,8,10)$ und in manchen Fällen eine Mischung beider Arten für verschiedene TC. Weitere qualitative und quantitative Prozessforschung wird uns helfen, diese Mechanismen besser zu verstehen und zu unterscheiden. Im Verlauf der Studie wurde während der Therapiephase eine grosse Menge qualitativer Daten gesammelt, einschliesslich des TherapeutInnen-Tagebuchs und der Audio- und Videoaufzeichnungen aller Sitzungen. Zukünftig werden wir die quantitativen Ergebnisse mit einer qualitativen Analyse der in unserer Studie gesammelten Daten kombinieren. Eine weitere Option für künftige Studien wäre es, die Prozessdaten bezüglich potenziellen Veränderungsmechanismen (z.B. Interventionen, um den Kontakt zu abgelehnten Gefühlen und Wünschen herzustellen) zu sammeln und Mediationsanalysen durchzuführen. All dies wird es ermöglichen, Ergebnisse zu erklären, die spezifischen Faktoren des GT-Ansatzes bei Angststörung $\mathrm{zu}$ identifizieren sowie die interne und externe Validität der Studie zu stärken.

Studiendesign: Trotz der aktuellen Beliebtheit der $\mathrm{N}=1$-Studien im Gesundheitswesen (Mengersen et al., 2011; Punja et al., 2016), gibt es Zweifel an ihrer internen und externen Validität, die untersucht werden müssen (Horner et al., 2005). Verzerrungen der Instrumente und Testmethoden wurden eliminiert, indem zuverlässige, gut erprobte Instrumente verwendet wurden. Der Behandlungsbias und die Bedrohung durch Reifeentwicklung (Maturation) wurden durch Arbeit an der dritten Forschungsfrage im Detail untersucht, wodurch eine natürliche Remission ausgeschlossen wurde. Es wird erwartet, dass andere therapieunabhängige Faktoren, die das Ergebnis beeinflussen, einschliesslich geschichtlicher Indikatoren (History), zukünftig durch ein Therapietagebuch aufgespürt werden. Die für die Experimentkontrolle schwierigste Bedrohung ist die Auswahlverzerrung, da es möglich ist, dass die Klientinnen, die sich freiwillig für die Studie gemeldet haben, bestimmte Eigenschaften teilen (z. B. Pflichtbewusstsein), die berücksichtigt werden müssen, um die Ergebnisse korrekt auswerten zu können. Die Gefahr des Stichprobenschwunds ist ebenfalls relevant und wir haben alle an der Studie beteiligten TherapeutInnen angewiesen, zu berichten, wenn ihre Klientinnen die Behandlung nicht abgeschlossen haben oder frühzeitig ausgeschieden sind.

Die externe Validität dieser Studie ist hoch, da die Fälle eine signifikant hohe Anzahl an Angststörungssymptomen und Begleiterkrankungen enthielten. Das Einzelfall-Zeitreihendesign erfüllt beide Anforderungsarten für externe Validität: Verallgemeinerbarkeit über die Situationen hinweg, aufgrund der direkten Verwendbarkeit der Studienergebnisse in realen Situationen in der Psychotherapie sowie Verallgemeinerbarkeit über die Menschen hinweg in dem Ausmass, dass alle PatientInnen mit einer allgemeinen Angststörungsdiagnose repräsentiert sind. Ein zusätzlicher Nutzen des Einzelfall-Zeitreihendesigns ist die Annäherung an den realen Therapieprozess, der im Umfang der ökologischen Validität eingeschlossen ist. Die ökologische Validität ist in unserer Studie von Natur aus vorhanden, im Gegensatz zu RCT, in der sie schwer $\mathrm{zu}$ erreichen ist.

Unsere Datenanalyse-Strategie berücksichtigt nahezu alle Vorschläge der Qualitätsstandards: die Anwendung visueller und statistischer Analysen; die Berücksichtigung der Autokorrelation; die Ergänzung der Zielbeschwerden mit Standard-Ergebnismessungen; die Prüfung auf therapieunabhängige Trends und Meta-Analyse (Borckardt $\&$ Nash, 2014; Borckardt et al., 2008; Kratochwill et al., 2013; Tate et al.; 2013; Wendt \& Miller, 2012). Die Verwendung des Glass' $\Delta$ hat für zukünftige Meta-Analysen jedoch Grenzen, da seine Auswertung offensichtlich nur für normalverteilte Daten (z.B. Fall 3) gedacht ist und unzureichende Werte ausgibt, wenn die Werte des Baseline-TC nur eine geringe oder keine Variation zeigen. Ausserdem war die Nutzung von SMA zur Kontrolle von therapieunabhängigen Trends nicht völlig zufriedenstellend. Die Begrenzung der Software von < 30 Datenpunkten pro Phase führte dazu, dass der Trend nur für die Baseline- und die Nachbeobachtungsphasen und nicht in der Therapiephase kontrolliert wurde, was zu Verzerrungen in den Berechnungen führen kann. Es ist sinnvoll, zukünftig das Package $\mathrm{R}$ anstelle einer SMA zu verwenden, um die dritte Forschungsfrage zu beantworten.

Einschränkungen und Vorschläge für die weitere Forschung: Wir müssen an dieser Stelle einige Einschränkungen unserer Studie und daraus resultierende Folgerungen für die weitere Forschung erwähnen: (1) In unserer Stichprobe waren Klientinnen mit unterschiedlichen Arten von Angststörungen. Obwohl dies der realen Situation im Praxisalltag entspricht und damit auch den Intentionen einer praxisorientierten Studie, würde eine differenziertere Stichprobenauswahl eine genauere Untersuchung der Veränderungsmechanismen bei GT für spezifische Angststörungssymptome ermöglichen. (2) Eine GT-Therapietreue-Skala, die sich jetzt, dank der gemeinsamen Bemühungen der internationalen GT-Gemeinschaft, 
in der Entwicklung befindet (Fogarty et al., 2016), war zum Zeitpunkt unserer Studie noch nicht verfügbar. Die Therapietreue wurde in unserer Studie auf andere Weise sichergestellt, aber für zukünftige Studien wird die GT-Therapietreue-Skala die erste Wahl sein. (3) Die TherapeutInnen waren Studierende eines Instituts vor dem Abschluss mit wenigen Jahren klinischer Praxis, dies stellt eine Einschränkung der externen Validität unserer Studie dar. (4) Die korrekte Formulierung der TC in Sitzung 0 ist von grösster Bedeutung. Ein Beispiel für die negativen Folgen eines Fehlers ist TC2 von Patientin 7: «Ich kann das Mobbing an meinem Arbeitsplatz nicht ertragen", das keine klinische Besserung aufwies, denn man kann hinterfragen, ob dies wirklich ein ökologisches Therapieziel war. Die Verschlechterung dieses TC könnte bedeuten, dass die Patientin sich das Mobbing nicht länger gefallen lässt und sich selbst schützt, oder es könnte bedeuten, dass sie weniger in der Lage ist, das Mobbing zu ertragen und daher stärker leidet. Um diese und andere mögliche Interpretationen zu klären, müssten an einer solchen Studie beteiligte TherapeutInnen besser in der Fähigkeit, TC zu formulieren, geschult sein. (5) Es gab nur unvollständige Daten über Menschen, die überlegten, eine Therapie zu beginnen, aber nicht an der Studie teilnehmen wollten, was sich auf die bereits genannten Auswahlverzerrungen und Verzerrungen durch Stichprobenschwund bezieht.

Die Konsequenzen für weitere Forschungsprojekte sind hauptsächlich zwei Strategien: Ausweitung des Projekts und Einbindung von sowohl Prozessergebnissen als auch qualitativen Forschungsergebnissen in ein komplexeres Studiendesign. Das Projekt kann auf verschiedene Arten ausgeweitet werden: (1) Es sollten TherapeutInnen mit unterschiedlicher praktischer Erfahrung eingeschlossen werden. (2) Durch die Nutzung des bereits errichteten GT-Forschungsnetzwerks können mit dieser Methode Fälle aus unterschiedlichen Ländern in die Studie eingeschlossen werden, wodurch die Meta-Analyse erweitert und bereichert werden würde. (3) Es können verschiedene Designs und alternative Instrumente angewendet und dabei das Einzelfall-Zeitreihendesign beibehalten werden, indem zum Beispiel EB-45 durch CORE-OM ersetzt wird. (4) Das Einzelfall-Zeitreihendesign kann für GT-Arbeit mit anderen Diagnosen verwendet werden, wie zum Beispiel bei Depression. (5) Die konstante Dokumentation der TC durch KlientInnen kann als achtsamkeitsfördernde Intervention untersucht werden.

Das Einzelfall-Zeitreihendesign kann nicht nur für Outcome-Studien nützlich sein. In diesem Artikel haben wir uns auf die Wirksamkeitsergebnisse und quantitative Methoden konzentriert. Wie wir jedoch kurz in der detaillierten Fallanalyse gezeigt haben, bietet das EinzelfallZeitreihendesign detaillierte und kontinuierliche Informationen zum Veränderungsprozess. Die Durchführung einer qualitativen Analyse und besonders die Untersuchung des Veränderungsmechanismus können die Ergebnisse ergänzen. Die Analyse der Videoaufzeichnungen der Sitzungen und der Folgeinterviews mit KlientInnen würde uns ermöglichen, die besten und schlechtesten Sitzungen sowie Wendepunkte während des Therapieprozesses zu verglei- chen und zu untersuchen, wie in diesen Fällen Änderungen eingetreten sind (vor allem bezüglich der Symptome der Angststörung). Qualitative Beobachtungen würden es uns ermöglichen, spezifische Veränderungsprozesse und damit zusammenhängende GT-Interventionen in den verschiedenen Fällen zu identifizieren, und diese mit KVT- und HE-Ansätzen zu vergleichen. Daten über nicht erfolgreiche Fälle können dabei helfen, die Grenzen der GT bei der Arbeit mit Angststörungen aufzuzeigen. Daraus ergeben sich auch Konsequenzen für die Psychotherapie-Praxis, denn das Einzelfall-Zeitreihendesign kann sinnvoll in einem Feedback-Informed Treatment (FIT) eingesetzt werden und ebenso als Lern- und Supervisionsmittel für unerfahrene TherapeutInnen.

Die praktischen Konsequenzen liegen nicht nur in der Empfehlung eines weiteren wirksamen PsychotherapieAnsatzes für KlientInnen mit Angststörungen, sondern auch in der Möglichkeit, das aufgestellte Studiendesign für psychotherapeutische Wirksamkeitsstudien in privaten Praxisumgebungen mit verschiedenen PatientInnengruppen zu verwenden. Einzelfall-Zeitreihendesigns können als sinnvolle Alternative von RCT eingesetzt werden, insbesondere in naturalistischen Outcome-Studien mit klaren Vorteilen für die Untersuchung des therapeutischen Veränderungsprozesses in praxisbasierten Forschungsnetzwerken.

\section{Danksagungen}

Wir möchten unseren Kollegen Jörg Bergmann, Otto Glanzer, Stefan Pfleiderer, Vincent Beja und Tomas Rihacek für ihre Hilfe in den verschiedenen Phasen dieser Studie danken.

\section{Literatur}

American Psychological Association [APA] (2006). Evidence-based practice in psychology. American Psychologist, 61(4), 271-285 (http://doi.org/10.1037/0003-066X.61.4.271).

Angus, L., Watson, J.C., Elliott, R., Schneider, K. \& Timulak, L. (2015). Humanistic psychotherapy research 1990-2015: From methodological innovation to evidence-supported treatment outcomes and beyond. Psychotherapy Research, 25(3), 330-347 (http://doi.org/10.1080/10503307.2014.989290).

Battle, C. C., Imber, S. D. \& Hoehn-Saric, R. (1966). Target complaints as criteria of improvement. American Journal of Psychotherapy, 20(1), 184-192.

Beck, A. T. (1978). Beck Depression Inventory. San Antonio/TX: Harcourt Brace Jocanocich.

Beretvas, S. N. \& Chung, H. (2008). A review of meta-analyses of single-subject experimental designs: Methodological issues and practice. Evidence-Based Communication Assessment and Intervention, 2, 129-141 (http://dx.doi.org/10.1080/17489530802446302).

Borckardt, J.J. \& Nash, M.R. (2014). Simulation modelling analysis for small sets of single-subject data collected over time. Neuropsychological Rehabilitation, 24(3-4), 492-506 (http://doi.org/10.108 0/09602011.2014.895390).

Borckardt, J.J., Nash, M.R., Murphy, M.D., Moore, M., Shaw, D. \& O’Neil, P. (2008). Clinical practice as natural laboratory for psychotherapy research: a guide to case-based time-series analysis. The American Psychologist, 63(2), 77-95 (http://doi.org/10.1037/0003066X.63.2.77). 
Campbell, J.M. (2003). Efficacy of behavioral interventions for reducing problem behavior in persons with autism: A quantitative synthesis of single-subject research. Research in Developmental Disabilities, 24, 120-138.

Carey, T.A. \& Stiles, W.B. (2015). Some Problems with Randomized Controlled Trials and Some Viable Alternatives. Clinical Psychology \& Psychotherapy, 23(1), 87-95 (http://doi.org/10.1002/ cpp.1942).

Ceballos, D. (2014). Trastorno De Ansiedad Generalizada. Figura Fondo, 36, 1-13.

Chambless, D. L., Baker, M.J., Baucom, D.H., Beutler, L.E., Calhoun, K.S., Crits-Christoph, P., ... Woody, S. (1998). Update on empirically validated therapies, II. Clinical Psychologist, 51(1), 3-16.

Chambless, D. L. \& Hollon, S.D. (1998). Defining empirically supported therapies. Journal of Consulting and Clinical Psychology, 66(1), 7-18.

Chambless, D.L. \& Ollendick, T.H. (2001). Empirically supported psychological interventions: controversies and evidence. Annual Review of Psychology, 52(1), 685-716.

Craske, M. G., Treanor, M., Conway, C. C., Zbozinek, T. \& Vervliet, B. (2014). Maximizing exposure therapy: An inhibitory learning approach. Behaviour Research and Therapy, 58, 10-23 (http://doi. org/10.1016/j.brat.2014.04.006).

Deane, F. P., Spicer, J. \& Todd, D. M. (1997). Validity of a Simplified Target Complaints Measure. Assessment, 4(2), 119-130 (http://doi. org/10.1177/107319119700400202).

Ecker, B., Ticic, R. \& Hulley, L. (2012). Unlocking the Emotional Brain. London: Routledge.

Elliot, R. (2003). CSEP-II Experiential Therapy Session Form. Toledo/ $\mathrm{OH}$ : University of Toledo Department of Psychology.

Elliott, R. (2013). Person-centered/experiential psychotherapy for anxiety difficulties: Theory, research and practice. Person-Centered $\mathcal{O}$ Experiential Psychotherapies, 12(1), 16-32 (http://doi.org/10.1080 114779757.2013.767750).

Elliott, R., Greenberg, L. S., Watson, J.C., Timulak, L. \& Freire, E. (2013). Research on Humanistic- Experiential Psychotherapies. In M. Lambert (Hrsg.), Bergin and Garfield's Handbook of Psychotherapy and Behavior Change (S. 495-538). New York: John Wiley \& Sons.

Foa, E. B. \& Kozak, M.J. (1986). Emotional processing of fear: exposure to corrective information. Psychological Bulletin, 99(1), $20-35$.

Fogarty, M., Bhar, S., Theiler, S. \& O'Shea, L. (2016) What do Gestalt therapists do in the clinic? The expert consensus. British Gestalt Journal, 25(1), 32-41.

Folke, F., Hursti, T., Tungström, S., Söderberg, P., Kanter, J. W., Kuutmann, K., ... Ekselius, L. (2015). Behavioral activation in acute inpatient psychiatry: a multiple baseline evaluation. Journal of Behavior Therapy and Experimental Psychiatry, 46, 170-181 (http:// doi.org/10.1016/j.jbtep.2014.10.006).

Francesetti, G., Gecele, M. \& Roubal, J. (Hrsg.). (2013). Gestalt therapy in clinical practice: From psychopathology to the aesthetics of contact. Milan: FrancoAngeli.

Glass, G. V., McGaw, B. \& Smith, M.L. (1981). Meta-analysis in social research. Beverly Hills/CA: Sage.

Greenberg, L.S. (1983). Toward a task analysis of conflict resolution in Gestalt intervention. Psychotherapy: Theory, Research, Practice, Training, 20(2), 190-201.

Hamilton, M. (1959). The assessment of anxiety states by rating. The British Journal of Medical Psychology, 32(1), 50-55.

Hague, B., Scott, S. \& Kellett, S. (2015) Transdiagnostic CBT Treatment of Co-morbid Anxiety and Depression in an Older Adult: Single Case Experimental Design. Behavioural and Cognitive Psychotherapy, 43(1), 119-124.

Herrera, P. (2016). The construction of a gestalt-coherent outcome measure. In J. Roubal, P. Brownell, G. Francesetti, J. Melnick \& J. Zeleskov-Djoric (Hrsg.), Towards a Research Tradition in Gestalt Therapy (S. 1-368). Newcastle upon Tyne: Cambridge Scholars Publishing.
Hollon, S.D. \& Beck, A. (2013). Cognitive and Cognitive-Behavioral Therapies. In M. Lambert (Hrsg.), Bergin and Garfield's Handbook of Psychotherapy and Behavior Change (S. 393-442). New York: John Wiley \& Sons.

Horner, R.H., Carr, E. G., Halle, J., Mcgee, G., Odom, S. \& Wolery, M. (2005). The use of single-subject research to identify evidence-based practice in special education. Exceptional Children, $71(2), 165-179$

Kratochwill, T.R. \& Levin, J.R. (2010). Enhancing the scientific credibility of single-case intervention research: Randomization to the rescue. Psychological Methods, 15(2), 124-144 (http://doi. org/10.1037/a0017736).

Kratochwill, T. R., Hitchcock, J.H., Horner, R.H., Levin, J. R., Odom, S.L., Rindskopf, D. M. \& Shadish, W.R. (2013). Single-case intervention research design standards. Remedial and Special Education, 34, 26-38.

Lambert, M. (2013). Bergin and Garfield's Handbook of Psychotherapy and Behavior Change. John Wiley \& Sons.

Lambert, M., Burlingame, G., Umphress, V., Hansen, N., Verneersch, D. \& Clouse, G. (1996) The Reliability and Validity of the Outcome Questionnaire. Clinical Psychology and Psychotherapy, 3, 106-116.

Manolov, R. \& Solanas, A. (2008). Comparing N = 1 effect sizes in presence of autocorrelation. Behavior Modification, 32, 860-875 (http://doi.org/10.1177/0145445508318866).

Manolov, R., Guilera, G. \& Sierra, V. (2014) Weighting strategies in the meta-analysis of single-case studies. Behavior Research Methods, 46, 1152 (https://doi.org/10.3758/s13428-013-0440-0).

Mengersen, K., McGree, J.M. \& Schmid, C.H. (2015) Systematic Review and Meta-analysis Using N-of-1 Trials. In J. Nikles \& G. Mitchell (Hrsg.), The Essential Guide to N-of-1 Trials in Health. Dordrecht: Springer (https://doi.org/10.1007/978-94).

Olive, M.L. \& Smith, B. W. (2005). Effect size calculations and single subject designs. Educational Psychology, 25(2-3), 313-324 (http:// doi.org/10.1080/0144341042000301238).

Perls, F. S. (1969). Gestalt Therapy Verbatim. New York: Bantam Books. Punja, S., Bukutu, C., Shamseer, L., Sampson, M., Hartling, L., Urichuk, L. \& Vohra, S. (2016). N-of-1 trials are a tapestry of heterogeneity. Journal of Clinical Epidemiology, 76, 47-56.

Robine, J.M. (2013). Anxiety Within the Situation: Disturbances of Gestalt Construction. In G. Francesetti, M. Gecele \& J. Roubal (Hrsg.), Gestalt Therapy in Clinical Practice. From Psychopathology to the Aesthetics of Contact (S. 479-495). Milano: FrancoAngeli.

Roth, A. \& Fonagy, P. (2013). What Works for Whom? Second Edition. New York: Guilford Press.

Roubal, J., Gecela, M. \& Francesetti, G. (2013). Gestalt Therapy Approach to Diagnosis. In G. Francesetti, M. Gecele \& J. Roubal (Hrsg.), Gestalt Therapy in Clinical Practice. From Psychopathology to the Aesthetics of Contact (S. 79-106). Milano: FrancoAngeli.

Shahar, B., Bar-Kalifa, E. \& Alon, E. (2017). Emotion-Focused Therapy for Social Anxiety Disorder: Results from a Multiple-Baseline Study. Journal of Consulting and Clinical Psychology, 85(3), 238249 (http://doi.org/10.1037/ccp0000166).

Sheehan, D., Lecrubier, Y., Harnett-Sheehan, K., Amorim, P., Janavs, J., Weiller, E., Hergueta, T., Baker, R. \& Dunbar, G. (1998). The MINI International Neuropsychiatric Interview (MINI): The Development and Validation of a Structured Diagnostic Psychiatric Interview. Journal of Clinical Psychiatry, 59(20), 22-33.

Silberschatz, G. (2017). Improving the yield of psychotherapy research. Psychotherapy Research, 27(1), 1-13 (http://doi.org/10.10 80/10503307.2015.1076202).

Smith, J.D. (2012). Single-case experimental designs: A systematic review of published research and current standards. Psychological Methods, 17(4), 510-550 (http://doi.org/10.1037/a0029312).

Smith, J.D., Borckardt, J.J. \& Nash, M.R. (2012). Inferential Precision in Single-Case Time-Series Data Streams: How Well Does the EM Procedure Perform When Missing Observations Occur in Autocorrelated Data? Behavior Therapy, 43(3), 679-685. 
Tate, R.L., Perdices, M., Rosenkoetter, U., Wakim, D., Godbee, K., Togher, L. \& McDonald, S. (2013). Revision of a method quality rating scale for single-case experimental designs and n-of- 1 trials: The 15-item Risk of Bias in N-of-1 Trials (RoBiNT) Scale. Neuropsychological Rehabilitation, 23(5), 619-638 (http://doi.org/10.10 80/09602011.2013.824383).

Timulak, L., McElvaney, J., Keogh, D., Martin, E., Clare, P., Chepukova, E. \& Greenberg, L. (2017). Emotion-Focused therapy for generalized anxiety disorder: An exploratory study. Psychotherapy, 54(4), 361-366.

Tschuschke, V., Crameri, A., Koemeda, M., Schulthess, P., Von Wyl, A. \& Weber, R. (2010). Fundamental reflections on Psychotherapy research and Initial results of the naturalistic Psychotherapy Study on outpatient treatment in Switzerland (PaP-S). International Journal of Psychotherapy, 14(3), 23-35.

von Bergen, A. \& de la Parra, G. (2002). OQ-45.2, Cuestionario para evaluación de resultados y evolución en psicoterapia: adaptación, validación e indicaciones para su aplicación e interpretación [OQ45.2, an outcome questionnaire for monitoring change in psychotherapy: adaptation, validation and indications for its application and interpretation]. Terapia Psicológica, 20(2), 162-176.

Watson, J. \& Greenberg, L. (2017). Emotion-Focused Therapy for Generalized Anxiety. Washington: APA Books.

Wendt, O. \& Miller, B. (2012). Quality Appraisal of Single-Subject Experimental Designs: An Overview and Comparison of Different Appraisal Tools. Education and Treatment of Children, 35(2), 235-268.

Whalon, K. J., Conroy, M. A., Martinez, J. R. \& Werch, B. J. (2015). SchoolBased Peer-Related Social Competence Interventions for Children with Autism Spectrum Disorder: A Meta-Analysis and Descriptive Review of Single Case Research Design Studies. J Autism Dev Disord, 45, 1513 (https://doi.org/10.1007/s10803-015-2373-1).

\section{«Evidence-based» as a criterion for psychotherapy selection? Concerning a good concept - and its misuse}

Despite the proven efficacy of CBT treatments for anxiety disorders, between $33 \%$ and $50 \%$ of patients do not respond or drop out of these treatments. Gestalt therapy has claimed to be an effective alternative, but there is little empirical evidence on its efficacy with anxiety. The Single-Case Experimental Design with Time Series Analysis was used as a practice-oriented study of efficacy. Evidence on ten clients diagnosed with anxiety disorders is presented, supporting the claim that Gestalt therapy can be a useful treatment for this. Detailed analysis of one case illustrates the changes in symptom and well-being scores, indicating turning points during the therapy. The paper discusses the use of this methodology for creating a practice-oriented research network.

Key words: Anxiety, Gestalt therapy, single-case, time series experimental design, practice-oriented research

\section{Examen de la gestalt-thérapie en cas de troubles de la peur dans des environnements pratiques. Un cas particulier de design expérimental}

Résumé : en dépit de l'efficacité prouvée de la thérapie du comportement cognitif (KVT) dans le cas des troubles de la peur généralisés, $33 \%$ des patientes et patients ne répondent pas à cette thérapie et $50 \%$ l'interrompent. La gestalt-thérapie est désignée comme une alternative efficace, mais il n'existe que peu de preuves empiriques de son efficacité dans le cas de troubles de la peur. Le design de cas particulier expérimental avec une analyse de série temporelle a été appliqué en tant qu'étude d'efficacité orientée vers la pratique. Les données de dix clientes présentant des troubles de la peur diagnostiqués y sont exposées, lesquelles viennent donner corps à l'affirmation en vertu de laquelle la gestalt-thérapie peut représenter un traitement utile pour cette indication. L'analyse détaillée d'un cas permet de visualiser la modification des scores pour les symptômes et le bien-être, en illustrant ainsi les points de retournement au cours de la thérapie. Ce papier traite de l'application de cette méthodologie dans le but de créer un réseau de recherche orienté vers la pratique.

Mots clés : trouble de la peur, gestalt-thérapie, cas particulier, design de série temporelle expérimentale, recherche orientée vers la pratique

\section{Ricerca sulla terapia della Gestalt nei disturbi d'ansia in contesti pratici. Un disegno sperimentale a caso singolo} Riassunto: Nonostante la comprovata efficacia della terapia cognitivo-comportamentale (CBT) nei disturbi d'ansia generalizzati, il $33 \%$ dei pazienti non risponde al trattamento e il $50 \%$ lo interrompe. La terapia della Gestalt è considerata un'alternativa efficace, ma ci sono poche prove empiriche della sua efficacia nei disturbi d'ansia. Il disegno sperimentale a caso singolo con un'analisi delle serie cronologiche è stato applicato come studio di efficacia orientato alla pratica. In esso vengono presentati dati provenienti da dieci pazienti con un disturbo d'ansia diagnosticato a supporto dell'affermazione secondo cui la terapia della Gestalt può essere un trattamento utile per questa indicazione. L'analisi dettagliata di un caso spiega la variazione nei punteggi di sintomi e benessere e illustra i punti di svolta nel corso della terapia. L'articolo discute l'applicazione di questa metodologia per creare una rete di ricerca orientata alla pratica.

Parole chiave: disturbo d'ansia, terapia della Gestalt, caso singolo, disegno sperimentale con serie cronologiche, ricerca orientata alla pratica

The original English version of this article can be seen in the online version of our Journal: https://doi.org/10.30820/16649583-2019-2-53b

\section{Die Autoren}

Pablo Herrera - Department of Psychology, Faculty of Social Sciences, University of Chile, Santiago, Chile

Illia Mstibovskyi - Southern Regional Gestalt Institute, Rostovon-Don, Russland

Jan Roubal - Department of Psychology, Faculty of Social Science, Masaryk University in Brno, Tschechische Republik Philip Brownell - Portland Gestalt Therapy Training Institute, Portland/Oregon, USA

Die Autoren sind Wissenschaftler, Psychotherapeuten und Trainer der Gestalttherapie. Sie koordinieren das internationale praxisbasierte Forschungsnetzwerk für das Einzelfall-Zeitreihen-Projekt.

\section{Kontakt}

Pablo Herrera Salinas

Escuela de Psicología, Universidad de Chile

E-Mail: pabloherreras@uchile.cl 